\title{
Elucidating the Molecular Anatomy of Acetyl-CoA Carboxylase in Brassica Rapa for Evolving Climate-Resilient Interventions to Minimize Carbon Footprints
}

\author{
Chinmaya Kumar Das ${ }^{1 *}$, Umasankar Nayak $^{2}$, Preetinanda Pati $^{3}$ \\ ${ }^{1}$ Department of Plant Breeding \& Genetics (AICRP on Rapeseed \& Mustard, Ranital), Odisha University of Agriculture \& \\ Technology, Bhubaneswar, India \\ ${ }^{2}$ Department of Entomology (RRTTS, Ranital), Odisha University of Agriculture \& Technology, Bhubaneswar, India \\ ${ }^{3}$ Department of Agronomy (AICRP on Rapeseed \& Mustard, Ranital), Odisha University of Agriculture \& Technology, Bhubaneswar, \\ India \\ E-mail: iitk.chinmaya@gmail.com
}

Received: 1 March 2021; Revised: 28 May 2021; Accepted: 25 June 2021

\begin{abstract}
Climate change is an emerging threat to food \& nutritional security. It adversely affects crop production by altering the gene expression patterns of genes encoding for growth, development, and crop yield. Further, carbon emissions during crop production processes coupled with rapid urbanization \& industrialization, and deforestation drive aggravate the climate change problem. Therefore, innovative adaptive measures must be developed in terms of climateresilient interventions for enhancing productivity by minimizing expanding carbon footprints. In this investigation, we developed molecular models of different components (biotin carboxylase (BC), biotin carboxyl carrier protein (BCCP), and carboxyl transferase (CT)) of an important enzyme acetyl-CoA carboxylase (ACC) of Brassica rapa which play a vital role in carbon sequestration in fatty acids and regulation of fatty acid metabolism. We successfully built molecular models of BC, BCCP, CT- $\alpha$, and CT- $\beta$ having sufficient degree of reliability and stereochemical quality scores as obtained from the structure validation using PROCHECK, ProSA, Verify3D, and ERRAT. Further, we did a set of molecular docking studies with models of BC, BCCP, and CT (CT- $\alpha$, CT- $\beta$ ) with their ligands (ATP, biotin, acetyl coenzyme-A) to know the active site residues involved in catalysis reaction using AutoDock-Vina. Our findings on the molecular architecture of different components of ACC in Brassica rapa and ligand binding sites of component proteins from the molecular docking studies will help in two different ways. Firstly, structural information of model would facilitate designing of site-directed mutagenesis based functional genomic studies for comprehending the putative role of ACC in fatty acid biosynthesis, regulation of ACC by light \& other molecular players reported in other species such as CTI \& PII proteins. Further, functional haplotype markers can be designed using active site information of ACC in Brassica rapa to improve oil content by amalgamating desired set of available genomic variations present in different cultivars and landraces using the molecular breeding programme and genome editing tools. Such findings are potential drivers for minimizing carbon footprints by sequestering carbon in carbon skeletons of fatty acids in minimal input requiring oil-producing crop plants (Brassica rapa).
\end{abstract}

Keywords: Brassica rapa, acetyl-CoA carboxylase, climate change

Copyright $(02021$ Chinmaya Kumar Das, et al

DOI: https://doi.org/10.37256/sce.222021803

This is an open-access article distributed under a CC BY license

(Creative Commons Attribution 4.0 International License)

https://creativecommons.org/licenses/by/4.0/ 


\section{Introduction}

Climate change is a serious concern which has evolved as a result of global warming attributed to excessive emission of greenhouse gases (GHGs) namely carbon dioxide, methane, nitrous oxide, fluorinated gases due to adverse anthropogenic activities including intensification of urbanization and industrial drive, deforestation and excessive burning of fossil fuels. GHGs emission simply expands the carbon footprints in the atmosphere. $\mathrm{CO}_{2}$ gas takes dominance out of all GHGs in terms of emission into the atmosphere due to different processes of the economic sector. Global emission of $\mathrm{CO}_{2}$ occurs mainly due to electricity \& heat production (25\%), industry (21\%), agriculture \& forestry-land usage system (24\%), transportation sector (14\%), buildings $(6 \%)$, and other energy sectors (10\%) due to burning of fossil fuels. ${ }^{1}$ Out of different sectors of the economy, agriculture and its associated sector also plays a major role in increasing carbon footprints in the atmosphere which simply thickens the atmospheric blanket causing global warming resulting in climate change. In this context, an increase in temperature of $0.5-1.2^{\circ} \mathrm{C}$ by $2020,0.88-3.16^{\circ} \mathrm{C}$ by 2050 , and $1.56-5.44^{\circ} \mathrm{C}$ by 2080 for the Indian region is predicted by Intergovernmental Panel on Climate Change (IPCC). ${ }^{2}$ The rising temperature trend due to climate change is going to adversely affect the agriculture sector. Under high-temperature conditions, metabolomic \& biochemical circuits of crop plants would not function in a normal way resulting in altered crop physiology which ultimately affects the crop yield both in terms of quantity \& quality.

In Indian conditions, rising population pressure demands food, nutrition, and livelihood security. Therefore, there is a need to mitigate the adverse impacts of climate change by ensuring food \& nutritional security in the climate change scenario without expanding the negative environmental footprints such as carbon footprints. Therefore, innovative ways can help in the sequestration of carbon to reduce the impact of climate change. Adoption of diversification in the cropping systems involving the cultivation of oilseed, legume, and cereal crops in a defined sequence in the rotation system to lower C-footprints in field crops is reported. Similarly, the inclusion of broadleaf crops in cereal-based rotation system in the crop diversification has been reported to increase energy use efficiency, ${ }^{3}$ decrease pest infestation, ${ }^{4}$ and lowering of carbon footprints to a significant extent due to reduced dependency syndrome on synthetic fertilizers. ${ }^{5,6}$

Concerning the agriculture sector, there is a need to develop climate-resilient crops having increased carbon use efficiency for minimizing carbon emissions and maximizing carbon sequestration processes in crop biomass as adaptation measures against climate change. As a mitigation measure, dependency on biofuel is gaining momentum as an alternative to petroleum-derived fuels. ${ }^{7,8}$ The use of biofuel crops can help in the sequestration of emitted carbons in carbon skeletons of lipids. Biodiesel is an important biofuel produced from oilseed species such as soybean (Glycine max), rapeseed (Brassica napus, Brassica rapa, and Brassica juncea), sunflower (Helianthus annuus), flax (Linum usitatissimum), and the African oil palm (Elaeis guineensis). ${ }^{9}$ Among different crops, Brassica oilseed crops are potential crops having a major role in the edible oil economy and can serve a role in biofuel generation. Further, these crops use a lesser amount of water and grown during the winter season in Indian conditions. Global warming \& increased aridity is going to impair the growth, phenology, crop yield, and oil yield in Brassica oilseed crops. These crops first capture carbon in their green leaves in terms of sucrose molecules through the process of photosynthesis. Further, sucrose undergoes a set of biochemical transformations to sequester carbon in the carbon skeletons of lipids. Broadly different molecular mechanisms and processes involved in carbon fixation in lipid molecules are described as follows:

\subsection{Conversion of $\mathrm{CO}_{2}$ into sucrose molecules}

This step involves the generation of reducing power \& ATP, and the release of oxygen molecules due to photolysis of water molecules in the light reaction of photosynthesis. In the dark reaction of photosynthesis, Ribulose-1,5bisphosphate (RuBP) carboxylase/oxygenase (Rubisco) drives the primary carbon fixation reaction by catalyzing the reaction between RuBP and $\mathrm{CO}_{2}$ to produce two molecules of 3-carbon compounds, 3-phosphoglycerate (3-PGA). These 3-PGA molecules are phosphorylated and reduced by the end products of light reactions of photosynthesis (ATP and Nicotinamide Adenine Dinucleotide Phosphate (NADPH)) to synthesize triose phosphates which serve as the source of carbon for starch synthesis in chloroplast and sucrose synthesis in the cytosol. Carbon in the form of di-hydroxy acetone phosphate (DHAP) is transported from chloroplast to cytosol through phosphate translocation. Synthesized sucrose is transported into different sink tissues through the phloem of the plant for further utilization. Cytosolic fructose-1,6bisphosphatase (FBPase) and sucrose phosphate synthase (SPS) plays a vital role in the modulation of the sucrose biosynthetic pathway. ${ }^{10}$ 


\subsection{Conversion of sucrose into fatty acids}

\subsubsection{Conversion of sucrose into Acetyl Coenzyme-A (acetyl CoA)}

In most oilseed crops, sucrose is imported into cytosol wherein Hexose-6-phosphate from sucrose metabolism makes entry into the glycolytic pathway to produce three carbon atom pyruvate molecules. Pyruvate dehydrogenase (PDH) helps in the conversion of pyruvate molecules into acetyl-CoA molecules which serve as a precursor molecule for the synthesis of Malonyl Coenzyme-A and fatty acids. ${ }^{47}$

\subsubsection{Synthesis of Malonyl Coenzyme-A (malonyl-CoA)}

Acetyl-CoA carboxylase enzyme (ACCase) catalyzes the reaction between acetyl-CoA, ATP, and $\mathrm{HCO}_{3}$ to produce malonyl CoA. ${ }^{47}$

\subsubsection{Conversion of Acetyl Coenzyme-A and Malonyl Coenzyme-A into fatty acids}

This step involves a series of condensation (between acetyl CoA and malonyl CoA) dehydration and reduction reactions in which three isoforms of ketoacyl-ACP synthase (KAS) cooperate to elongate acyl chains on an ACP platform to $\mathrm{C} 16$ or C18. KASIII plays a role in the initiation of fatty acid synthesis by condensing acetyl CoA and malonyl CoA, KASI help in the elongation step up to C16:0. Similarly, KASII plays a role in the final elongation step from C16:0 to C18:0. Similarly, stearoyl-ACP desaturase (SAD) helps in the desaturation of 18:0 ACP to produce 18:1 ACP. Fatty acids are synthesized in the stroma of the plastid. In plants, fatty acid synthase consists of an acyl carrier protein and an enzyme. ${ }^{47}$

\subsubsection{Release of fatty acids from ACP for triacylglycerol (TAG) or lipid droplet synthesis in the endoplasmic reticulum}

Newly synthesized fatty acids namely C16:0, 18:0, C18:1 are released from the ACP in stroma by the action of thioesterase (FATA \& FATB) and are exported to the endoplasmic reticulum for TAG formation and lipid droplet formation. $^{47}$

Among different molecular players involved in the fatty acid biosynthesis, acetyl-CoA carboxylase (ACC) is one of the most important candidate players which catalyzes the first regulated \& rate-limiting step in the fatty acid synthesis. $\mathrm{ACC}$ is a multi-functional biotin-dependent carboxylase enzyme composed of three distinct components namely biotin carboxylase $(\mathrm{BC})$, biotin carboxyl carrier protein $(\mathrm{BCCP})$, and carboxyltransferase $(\mathrm{CT}) .^{11}$ Further, the carboxyl transferase subunit is composed of two subdomains namely CT- $\alpha$ and CT- $\beta .^{12}$ Broadly, two different half-reactions are catalyzed by ACC. Both BC and BCCP play a role in the first reaction to form carboxybiotin. Similarly, in the second half-reaction, carboxyltransferase $(\mathrm{CT})$ helps in the transfer of the carboxyl group from the carboxybiotin to acetyl-CoA to form malonyl-CoA. In the first half-reaction, biotin carboxylase (BC) catalyzes ATP-dependent carboxylation of biotin to form carboxybiotin, while both BCCP and CT participated in the second half reaction. ${ }^{13}$

Many scientific reports implicated the potential role of acetyl-CoA carboxylase as a potential player which could play a role in carbon fixation. The role of ACCase in the modulation of de novo fatty acid biosynthesis in Escherichia coli, yeast, and various mammalian sources is reported by many research groups. ${ }^{14}$ Overexpression of four different subunits of ACCase in Escherichia coli is found to increase the ACCase activity with the concomitant increase in the rate of fatty acid biosynthesis. ${ }^{15}$ Further, Menendez et al. ${ }^{16}$ proposed the role of acetyl-CoA carboxylase in autotrophic carbon fixation. Three major players have been reported in literature namely carboxyltransferase interactors (CTIs), biotin/lipoyl attachment domain-containing (BADC) proteins, and PII proteins influence ACC activity resulting in the modulation of lipid levels in plants. Light-mediated enhanced interaction between carboxyltransferase interactors (CTIs) and $\alpha$-carboxyltransferase $(\mathrm{CT}-\alpha)$ subunit of ACC cause attenuation of carbon flux into fatty acid biosynthesis and knockout of CTIs resulted in enhanced triglycerides in leaves in Arabidopsis thaliana. ${ }^{17}$ 18:1-acyl carrier protein plays a role in the feedback regulation of plastidial ACC in Brassica napus. ${ }^{18}$ Similarly, the interaction between BCCP subunit of ACC and a signal integrator protein PII modulates the feedback inhibition of plastidial ACC by reducing its Vmax. ${ }^{19}$ in Arabidopsis. Further, BADC (biotin/lipoyl attachment domain)-containing proteins play a role in the dark-light modulation of ACCase activity. ${ }^{17,20}$ Cloning and gene expression patterns of chloroplast-encoded CT- $\beta$ and BCCP genes 
in Brassica napus revealed their differential expression behavior during embryo development and in different tissues. ${ }^{21}$ Beta-carboxyltransferase(CT- $\beta$ ) (BnaC05g37990D) and biotin carboxyl carrier protein1 (BCCP1) (BnaA03g06000D) of heterogeneous ACCase play a role in the modulation of de novo fatty acid synthesis in Brassica napus. ${ }^{22}$ Transcript profiling analysis of 32 principal genes involved in the modulation of lipid biosynthesis revealed down-regulation of 10 genes (KAS3, CT- $\beta$, BcRK6, P450, FatA, Oleosin, FAD6, FatB, CT- $\alpha$, and SUC1) including two genes encoding for different components of ACC namely (CT- $\beta$ and CT- $\alpha$ ) by 20 to $50 \%$ in their transcriptional levels in Brassica napus. ${ }^{23}$ Mostly, genetic and gene expression studies on ACC are reported in the literature. The complete structural information of ACC is still not known in any organisms because of its structural complexity with its four subunits with different functions of each subunit. BC subunit plays a role in carboxylation while CT- $\beta$ plays a role in carboxyltransferase activity. Similarly, BCCP and CT- $\alpha$ plays a role in the regulation of ACC activity by interacting with downstream effectors. Further, most of the reported studies on ACC activity are reported in microbes \& Arabidopsis thaliana while few studies are reported in the oilseed crop Brassica napus. Very little is known on the role of ACC in fatty acid biosynthesis and its three-dimensional structural information in Brassica rapa which is a short-duration oilseed crop grown primarily during the winter season in India. Therefore, the development of the structural model of ACC in Brassica rapa using computational tools would further help in comprehending the putative role of ACC in fatty acid biosynthesis and the sequestration of carbon in carbon skeletons of fatty acid.

In this manuscript, an attempt has been made to understand the molecular architecture of different components of the acetyl-coenzyme carboxylase enzyme of Brassica rapa (Br_ACC) using structure-based investigations, homology modeling, and molecular docking studies. Findings from this investigation will shed light on designing climate-resilient interventions for minimizing carbon footprints by the sustainable sequestration of carbon in the carbon skeleton of fatty acids.

\section{Materials and methods}

\subsection{Sequence retrieval}

Amino acid sequences of following proteins corresponding to biotin carboxylase (XP_009118263.1[Brassica rapa]), biotin carboxyl carrier protein (XP_009121569.1[Brassica rapa], Q42533[Arabidopsis thaliana], G4WXE1[Brassica napus], G4WXE7[Brassica oleracea], G4WXD3[Brassica campestris] and P0ABD8[Escherichia coli]), $\alpha$ subunit of carboxyl transferase(XP_009143428.1 [Brassica rapa]), $\beta$ subunit of carboxyltransferase (ABZ10594.1[Brassica rapa]) of acetyl-CoA carboxylase from different species were retrieved from the protein database of NCBI (https://www.ncbi.nlm.nih.gov/protein). Retrieved amino acid sequences of BC, BCCP, and CT subunits of ACC of Brassica rapa were used as "target" proteins for the development of their three-dimensional models and from other species were used in multiple sequence alignment studies.

\subsection{Construction of three-dimensional models using homology modelling}

The three-dimensional model of different components of ACC in Brassica rapa namely biotin carboxylase (Br $\mathrm{BC})$, biotin carboxyl carrier protein $\left(\mathrm{Br} \_\mathrm{BCCP}\right)$, and carboxyltransferase [ $\alpha$-subunit of carboxyl transferase $\left(\mathrm{Br} \_\mathrm{CT}-\right.$ $\alpha$ ) and $\beta$-subunit of carboxyltransferase $\left(\mathrm{Br} \_\mathrm{CT}-\beta\right)$ ]were constructed from their amino acid sequences as "targets" using known structural information of biotin carboxylase from Staphylococcus aureus complexed with AMP-PNP and two $\mathrm{Mg}^{2+}$ ions (2vpq.1.A.pdb) ${ }^{24}$, E. coli_BCCP protein ${ }^{13}$ and structure of carboxyl transferase subunit of ACC from Staphylococcus aureus (2F9I.1.pdb), respectively as the structural template. All the models were constructed using the homology modeling-based approach with the help of SWISS-Model (https://swissmodel.expasy.org/) $)^{25}$ web server.

\subsection{Evaluation of built three-dimensional models}

All the built models were subjected to evaluation of the correctness of their structural descriptors and stereochemical parameters and the best models were selected. Following analysis were conducted for the validation of built models: 


\subsubsection{Protein structure analysis (ProSA)}

Protein structure analysis of built models was validated using ProSA-web server (https://prosa.services.came. sbg.ac.at/prosa.php). ProSA analysis is based on the atomic coordinates of the built model. Such analysis aids in the evaluation of the accuracy of the built models by computing their $\mathrm{C} \alpha$ potentials and energy of structures to produce two distinct outputs namely z-scores and residues energy plots. The z-score is a measure of the overall quality of the model which measures the deviation of the total energy of specified model structure concerning energy distributions derived from random conformations. ${ }^{26,27}$ In erroneous structures, z-scores will be in the outside range of native protein structures. Similarly, the energy plot of the model reflects the local quality of the model by plotting energies as a function of amino acid sequence position in which erroneous regions will have positive energies.

\subsubsection{PROCHECK}

Stereochemical quality of built models was analyzed for residue-by-residue geometry and overall structure geometry ${ }^{28}$ by inspecting the accuracy of their dihedral angles $(\varphi$ and $\psi$ ) in a graphical plot called Ramachandran plot.

\subsubsection{VERIFY3D}

Assessment of built atomic model (3D) with its amino acid sequence (1D) by assigning a structural class based on its location and environment and comparing the results to good structures was carried out using VERIFY3D tools (https:// saves.mbi.ucla.edu/). ${ }^{29}$

\subsubsection{ERRAT}

It helps in the examination of nonbonded atomic interactions to determine the overall quality of the model. A higher score from ERRAT represents a more native-like model..$^{30}$

\subsection{Molecular docking studies}

A set of molecular docking studies between the predicted 3D-model of different components of ACC in Brassica rapa (Br_BC, Br_BCCP, Br_CT- $\alpha$, Br_CT- $\beta$ ) and their putative ligands were carried out using AutoDock-Vina. ${ }^{31}$ Docking of Br_BC with ATP molecule, biotin with BCCP, acetyl-CoA, and biotin with $\beta$ and $\alpha$ subunit of CT domain, respectively, were carried out. The ligand molecules were downloaded from the PubChem database. ${ }^{32}$

\subsection{Visualization of 3D models and protein-ligand interactions}

Molecular architecture of built 3D-models of different components of acetyl-CoA carboxylase, and interaction between models \& their ligands were visualized using UCSF Chimera ${ }^{33}$ and with the help of an open-source graphic tool PyMol. ${ }^{34}$

\subsection{Multiple sequence alignment}

Multiple sequence alignment for biotin carboxylase, biotin carboxyl carrier protein, $\beta$ and $\alpha$ subunits of carboxyltransferase domain of ACC from different species were carried out using Clustal ${ }^{35}$ and were visualized using a multiple sequence alignment editor Jalview. ${ }^{36}$

\section{Results and discussion}

\subsection{Structural modelling of Biotin carboxylase $(B C)$ subunit of $A C C$ of Brassica rapa $\left(B r \_B C\right)$}

The 3D model of target Br_BC protein was constructed from the amino acid sequence of Br_BC(XP_009118263.1) retrieved from the NCBI-protein database using acetyl-CoA carboxylase crystal structure of biotin carboxylase from S. aureus complexed with AMP-PNP and two $\mathrm{Mg}^{2+}$ ions (2VPQ.1.A. pdb) as the template. ${ }^{24}$ The constructed 3D model of 
Br_BC was evaluated for different stereochemical and structural quality parameters using a set of structural validation tools namely PROCHECK ${ }^{28}$, ProSA ${ }^{26,27}$, VERIFY3D ${ }^{29}$ and ERRAT ${ }^{30}$. Such analysis indicated built 3D model of Br_BC has a sufficient score for accuracy and reliability. PROCHECK analysis revealed that Br_BC 3D model has sufficient stereochemical quality as it occupies more than $99 \%$ residues in the allowed region of Ramachandran plot $(90.5 \%$ in most favored core region, $9.0 \%$ in additional allowed region) and only $0.5 \%$ residues in the disallowed region (Table 1 ) (Figure 1 and Figure 2).

(a)

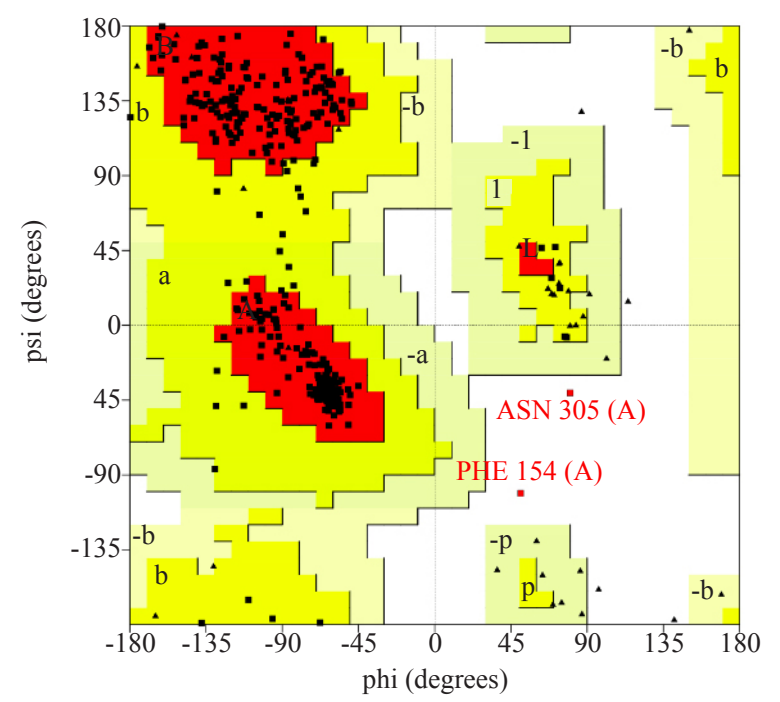

(c)

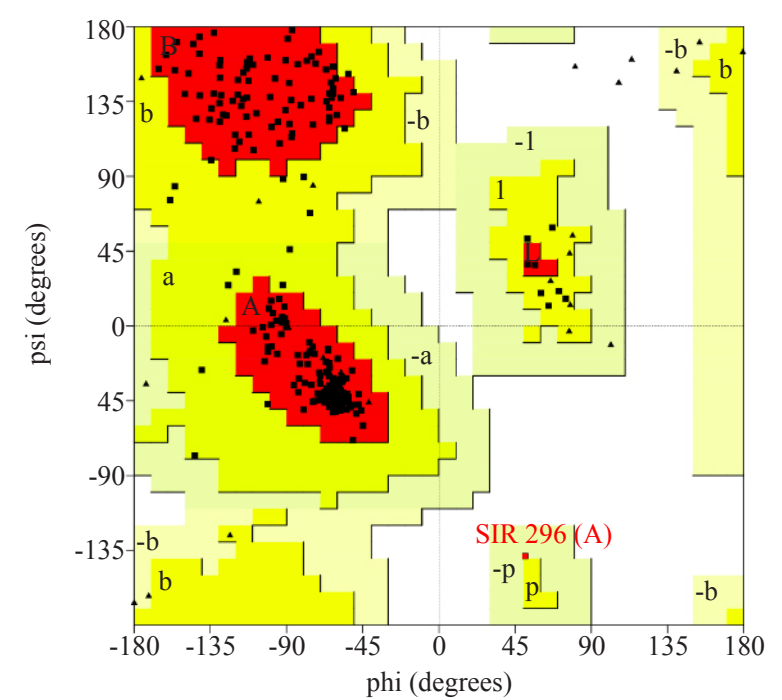

(b)

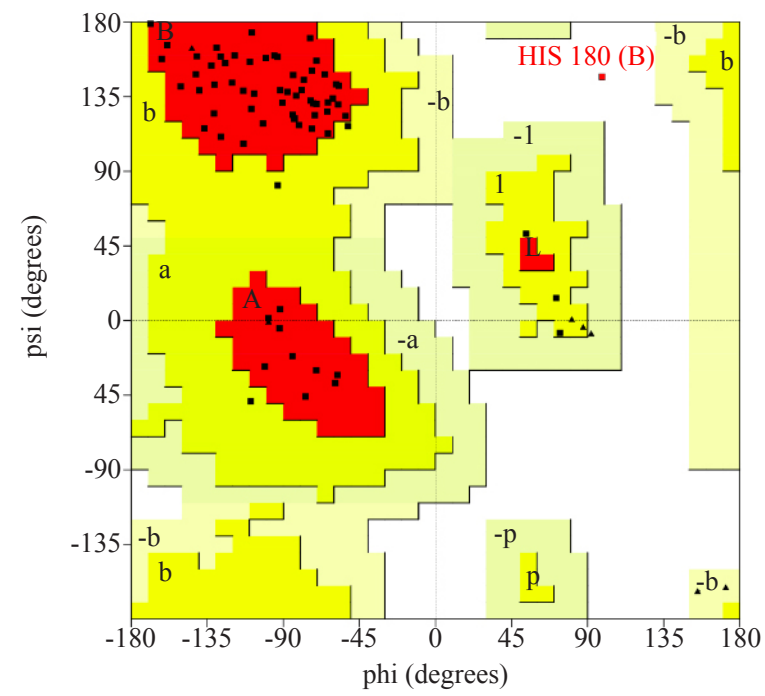

(d)

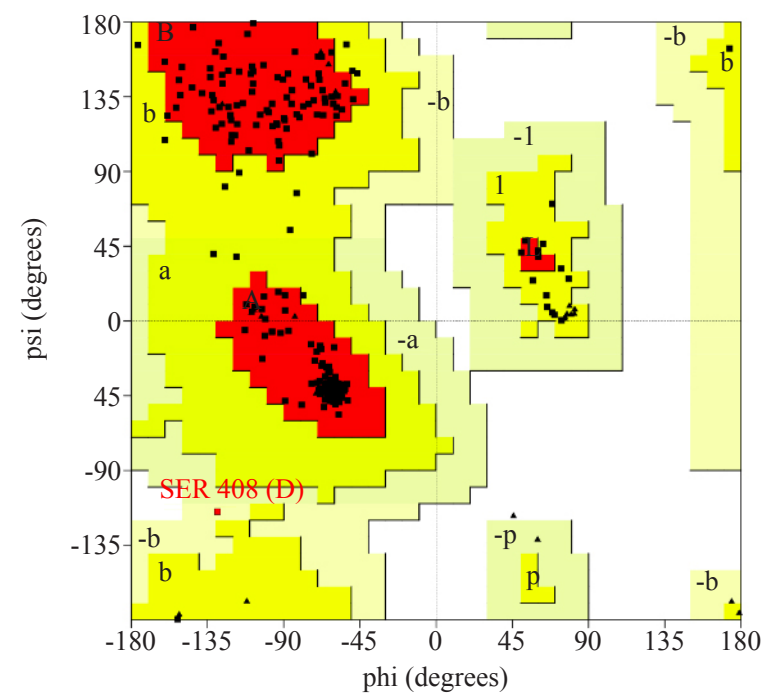

Figure 1. Representation of stereochemical quality of model using PROCHECK tools: Ramachandran plots of Br_BC, Br_BCCP, Br_CT- $\alpha$, and Br_ CT- $\beta$ are depicted in (a), (b), (c) and (d) panel of Figure 1, respectively to analyze the stereochemical quality of models.

Biotin carboxylase protein is a member of the ATP-grasp family and has a dependency on divalent cations like $\mathrm{Mg}^{2+}$ to modulate its ATP activity. ${ }^{37,38}$ The modeled structure of $\mathrm{Br}$ BC is composed of three distinct sub-domains (A-sub-domain at N-terminal, B-sub-domain, and C-subdomain at C-terminal) which is in line with its template. The A-subdomain of the model is present in between ASP73 to ASN176 residues and is consisted of four strands of a 
parallel $\beta$-pleated sheet flanked on either of its sides by a total of four $\alpha$-helices depicted in light-yellow color.
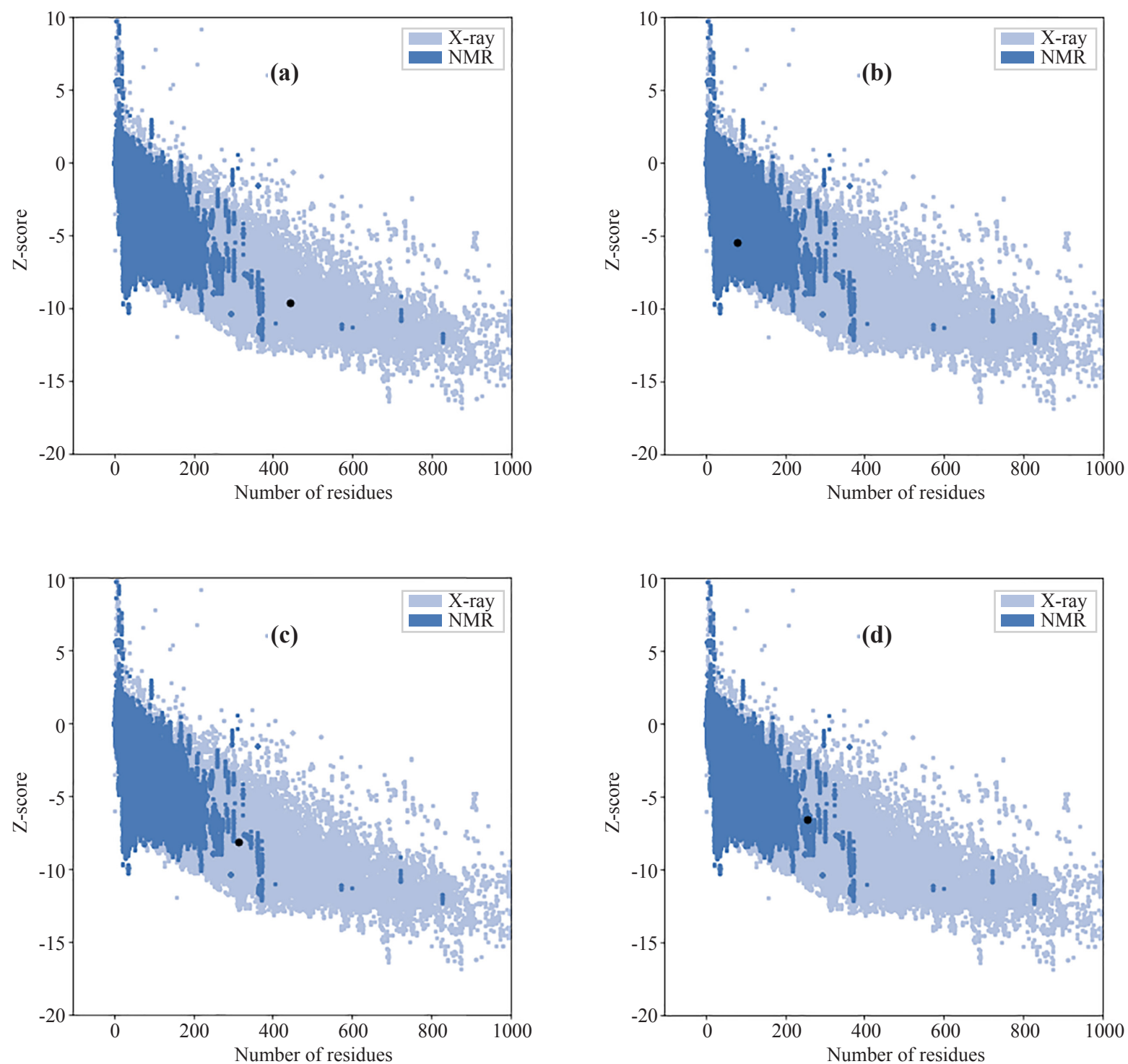

Figure 2. Representation of global quality of model using ProSA tools: Z-score plots of Br_BC, Br_BCCP, Br_CT- $\alpha$ and Br_CT- $\beta$ are depicted in (a), (b), (c), and (d) panel of Figure 2, respectively to analyze the global quality of models.

Table 1. Statistics related to validation of developed 3D models

\begin{tabular}{cccccccc}
\hline & $\begin{array}{c}\text { Core } \\
(\%)\end{array}$ & $\begin{array}{c}\text { Allowed } \\
(\%)\end{array}$ & $\begin{array}{c}\text { Gen. Allowed } \\
(\%)\end{array}$ & $\begin{array}{c}\text { Disallowed } \\
(\%)\end{array}$ & $\begin{array}{c}\text { PROSA } \\
\text { Z-Score }\end{array}$ & $\begin{array}{c}\text { Verify3D } \\
(\%)\end{array}$ & $\begin{array}{c}\text { ERRAT } \\
(\%)\end{array}$ \\
\hline Br_BC & 90.5 & 9.0 & 0.0 & 0.5 & -9.6 & 99.7 & 95.83 \\
Br_BCCP & 90.3 & 8.1 & 0.0 & 1.6 & -5.46 & 95.0 & 88.8 \\
Br_CT- $\alpha$ & 92.2 & 7.4 & 0.4 & 0.0 & -8.4 & 85.94 & 94.4 \\
Br_CT- $\beta$ & 89.6 & 10.0 & 0.5 & 0.0 & -6.56 & 71.2 & 89.4 \\
\hline
\end{tabular}


The B-subdomain is delineated by PRO177 to GLN274 residues present in the core region of the protein folded into three $\alpha$-helices and a $\beta$-pleated sheet of three strands depicted in hot pink color. Both A-subdomain and B-subdomain are connected by an AB-linker region shown in green color. After the B-subdomain, the polypeptide of the chain is folded into the body in the form of a $\mathrm{C}$-terminal domain consisting of a $\beta$-pleated sheet surrounded by four $\alpha$-helices (depicted in purple color) (Figure 3).Overall, both A and C subdomains form the body of the Br_BC protein while the B-subdomain forms the lid or head of this protein.

(a)

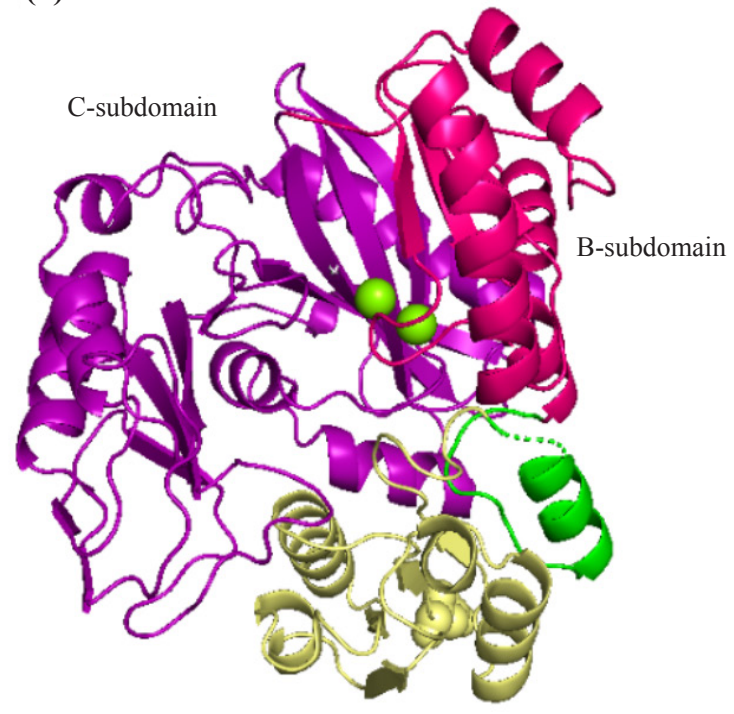

(b)

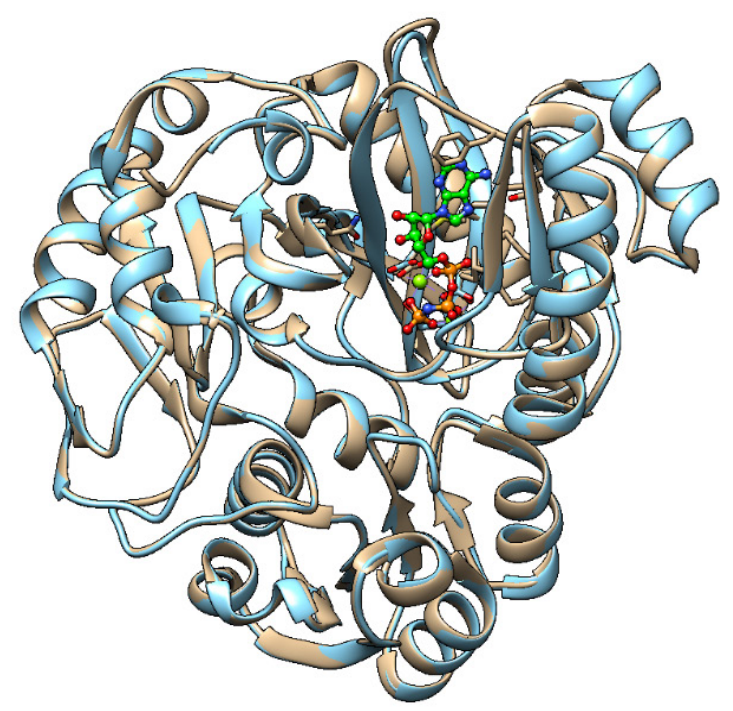

Figure 3. Depiction of the molecular architecture of biotin carboxylase domain of ACCase in Brassica rapa (Br_BC): (a)The three subdomains of Br $\mathrm{BC}$ are depicted in three different colors namely light yellow (for A-subdomain), hot pink color (for B-subdomain), and purple color (for C-subdomain). $\mathrm{AB}$-linker region is depicted in green color. Further, two green spheres representing two divalent magnesium cations are also depicted in the active site of the Br_BC model. (b) Superimposition structure of the backbone region of the template (grey color) and Br_BC model (cyan) color is shown. In addition, ATP and magnesium ions (green spheres) complexed in the active site of the template have also been depicted.

\subsubsection{Analysis of active site of biotin carboxylase subunit of ACCase in Brassica rapa revealed evolutionary conservation of residues involved in the binding of ATP}

Biotin carboxylase is involved in the ATP-dependent carboxylation of biotin. ${ }^{39}$ Therefore, to understand the binding site of ATP in Br_BC, a molecular docking study was carried out using AutoDock-Vina tools. ${ }^{31}$ Earlier, the significance of B and $\mathrm{C}$ subdomains for ATP interaction has been reported. ${ }^{24,40}$ In this investigation, ATP was docked at the earlier proposed binding site in $\mathrm{BC}$ and the stable docked conformation pose with minimal energy at the active site was selected for further analysis. Mapping of important residues within $4 \AA$ of the predicted ATP-binding active site was selected due to their functional significance in biological interaction. The residues L160, A159, N158, E157, F154, G153, Y152, R384, E360, R89, E93, R86, Y393, Q364, R437, S375, F371, E366, L455, S454, E412, R408, K307, Q306, F418, R303, R304, N305, M357, M238, G235, G234, G233, G232, G184, F262, and N360 were found to be present within a distance of $4 \AA$ from ATP in the active site. The oxygen atom of Q306 forms a salt bridge with the nitrogen atom of

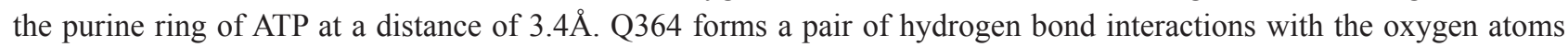
of ribose moiety of ATP at a distance of $2.2 \AA$ and $2.3 \AA$, respectively. K307 forms a hydrogen bond interaction with the oxygen atom of ribose moiety of ATP at a distance of $2 \AA$. N410 forms a pair of stabilizing hydrogen bond interactions with the gamma phosphate group of ATP at a distance of $2.7 \AA$ and $2.4 \AA$, respectively. 

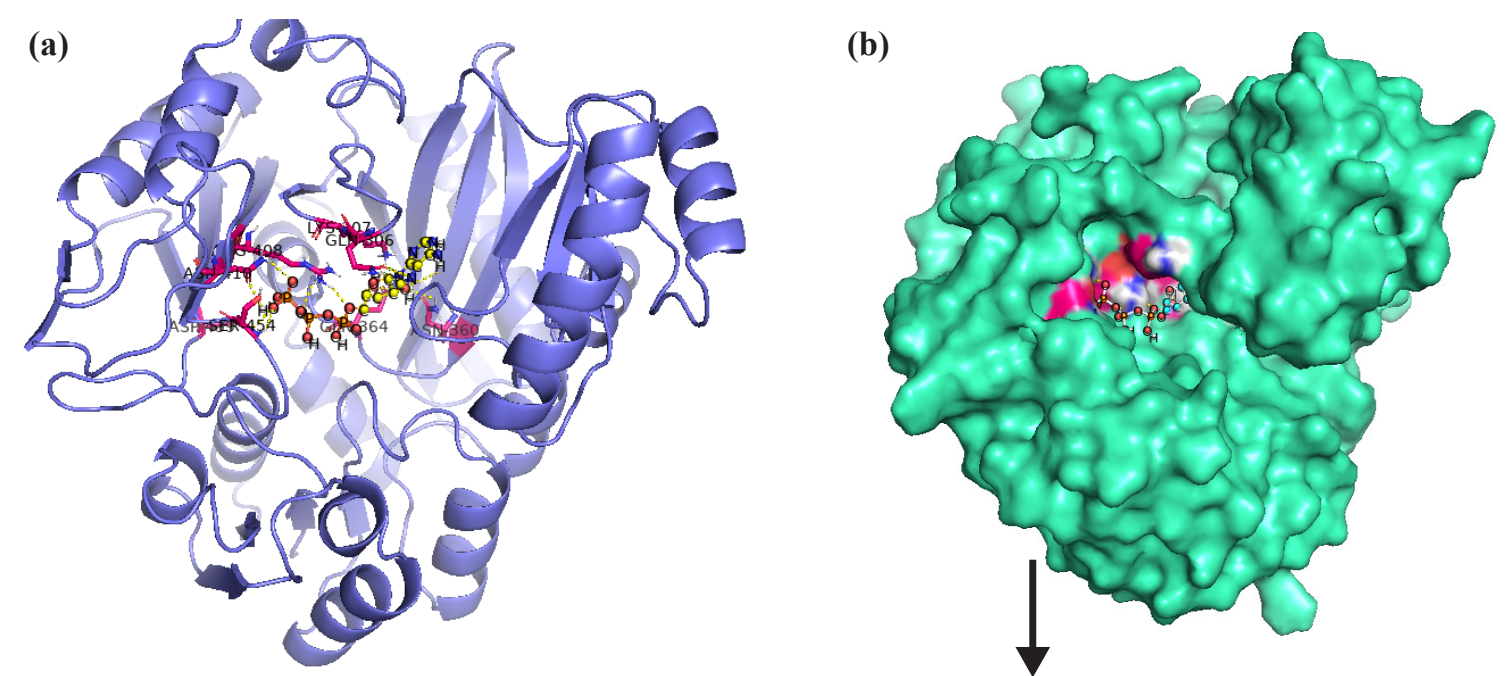

(c)
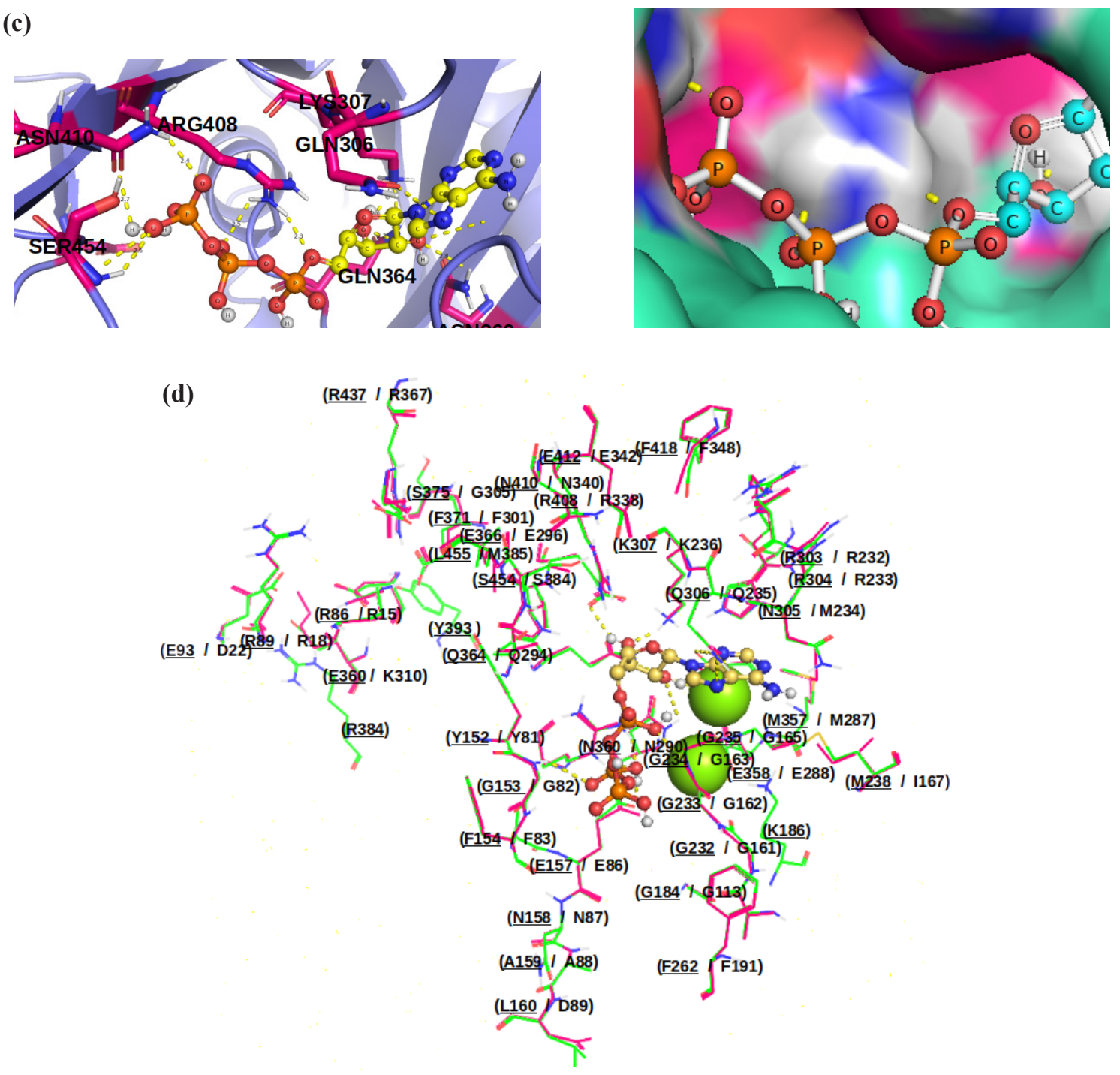

Figure 4. Visualization of molecular anatomy of the active site of biotin carboxylase component ACCase in Brassica rapa (Br BC): (a) Modeled structure of Br_BC docked with ATP is shown; (b) The surface view of Br BC with docked ATP in the active site is highlighted; (c) Key active site residues in $\mathrm{Br}$ BC model interacting with ATP in docked conformation is visualized; (d) Superposition of active site residues interacting with ATP of Br_BC model (shown in green color) with the template crystal structure of biotin carboxylase from S. aureus complexed with AMPPNP and two $\mathrm{Mg}^{2+}$ ions (2VPQ.1.A. pdb) (shown in pink color) is depicted. 
Similarly, S454 and L455 form a pair of stabilizing hydrogen bond interactions with oxygen atoms of the gamma phosphate group of ATP at a distance of $2.5 \AA$ and $2.8 \AA$, respectively. Similarly, N360 forms a hydrogen bond interaction with the oxygen atom of ribose moiety of ATP at a distance of $2.8 \AA$. Further comparison of active sites of Br_BC model and template revealed the conservation of most of the key residues except for few residues. Therefore, the potential biological role of variant active site residues can help in designing interventions to modulate fatty acid metabolism using chemical genetics and computer-aided designing approaches (Figure 4 and Figure 5).

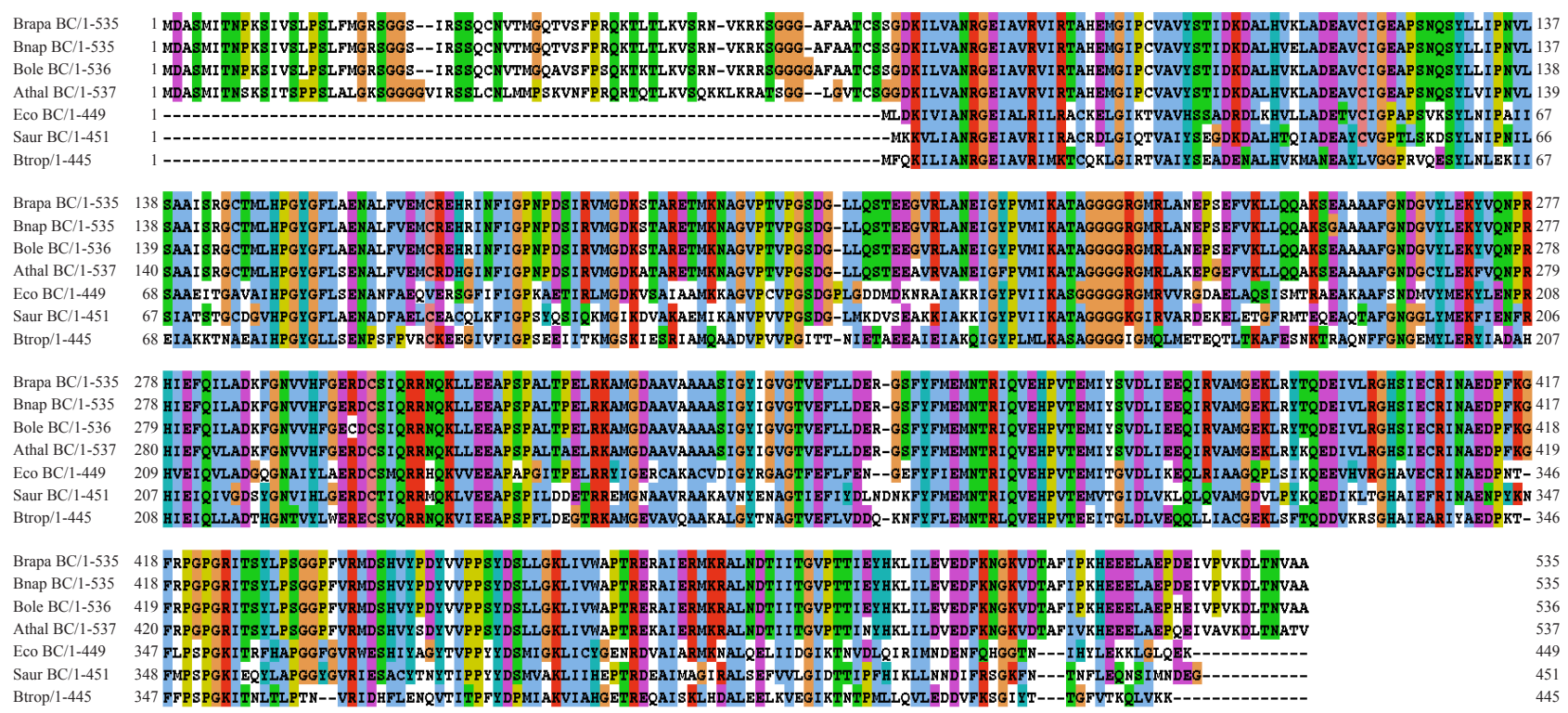

Figure 5. Multiple sequence alignment of biotin carboxylase subunit of ACCase: Biotin carboxylase subunit of different Brassica species along with Arabidopsis \& other microbial species is depicted in a. Br: Brassica rapa, Bn: Brassica napus, Bo: Brassica oleracea, At: Arabidopsis thaliana, Ec: Escherichia coli multiple sequence alignments, Saur: Staphylococcus aureus, Btrop: Bacillus tropius.

\subsection{Three-dimensional model of Brassica rapa BCCP $\left(\mathrm{Br}_{-} \mathrm{BCCP}\right)$}

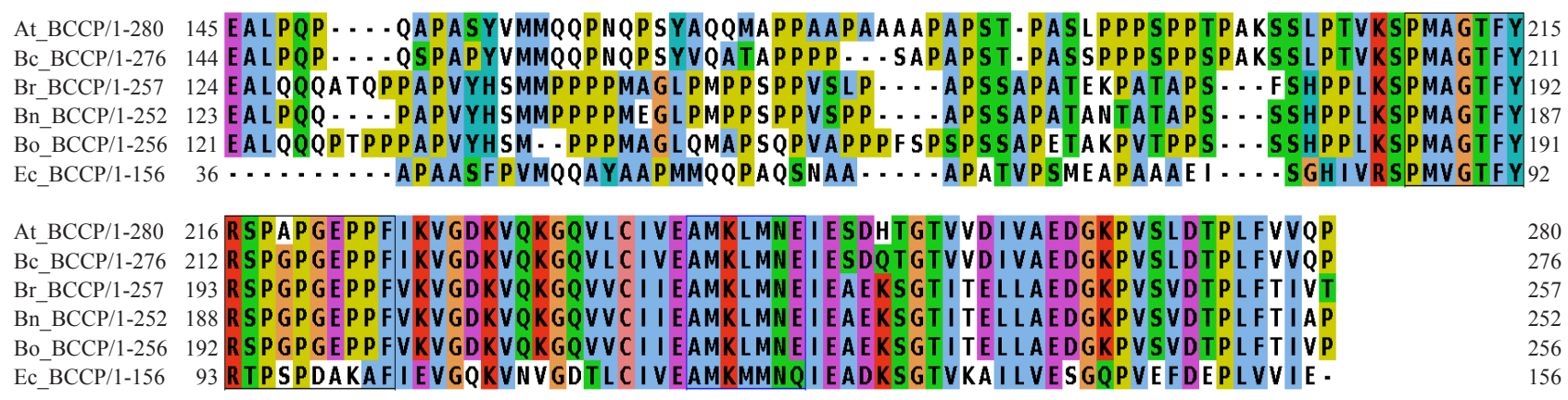

Figure 6. Multiple sequence alignment of biotinyl domain of BCCP: Biotinyl domain of different Brassica species along with Arabidopsis \& E.coli is represented in the MSA: At: Arabidopsis thaliana, Bc: Brassica campestris, Br: Brassica rapa, Bn: Brassica napus, Bo: Brassica oleracea, Ec: Escherichia coli

The 3D model of Br_BCCP was constructed from its amino acid sequences using the BCCP protein of E. coli as a template. ${ }^{13}$ The constructed 3D model of Br_BCCP was analyzed for different stereochemical and structural quality parameters using a set of structural validation tools namely PPROCHECK ${ }^{28}, \operatorname{ProSA}^{26,27}, \mathrm{VERIFY}^{29}$ and ERRAT $^{30}$. 
Such analysis indicated built 3D model of Br_BCCP has a sufficient score for accuracy and reliability. PROCHECK analysis revealed that Br_BCCP 3D model has sufficient stereochemical quality as it occupies more than $98 \%$ residues in the allowed region of Ramachandran plot (90.3\% in most favored core region, $8.1 \%$ in additional allowed region) and only $1.6 \%$ residues in the disallowed region (Table 1) (Figure 1 and Figure 2).

The modeled protein Br_BCCP is composed of two similar $\beta$-sheets each containing four $\beta$-strands and two $\beta$-sheets are very compactly arranged around a hydrophobic core which is a canonical and conserved feature in the biotin and lipoyl enzyme superfamily. ${ }^{41}$ The hydrophobic core of Br_BCCP biotinyl domain mainly harbours Pro186, Met187, Ala188, Gly189, Thr190, Phe191, Tyr192, Arg193, Ser194, Pro195, Gly196, Pro197, Gly198, Glu199, Pro200, Pro201, Phe202, Val203, Lys204, and Val205 residues which help in the stabilization of $\beta$-sandwich conformation ${ }^{42}$ and most of the residues in the hydrophobic core are evolutionarily conserved with high sequence identity among its homologs species as depicted in the multiple sequence alignment (Figure 6).
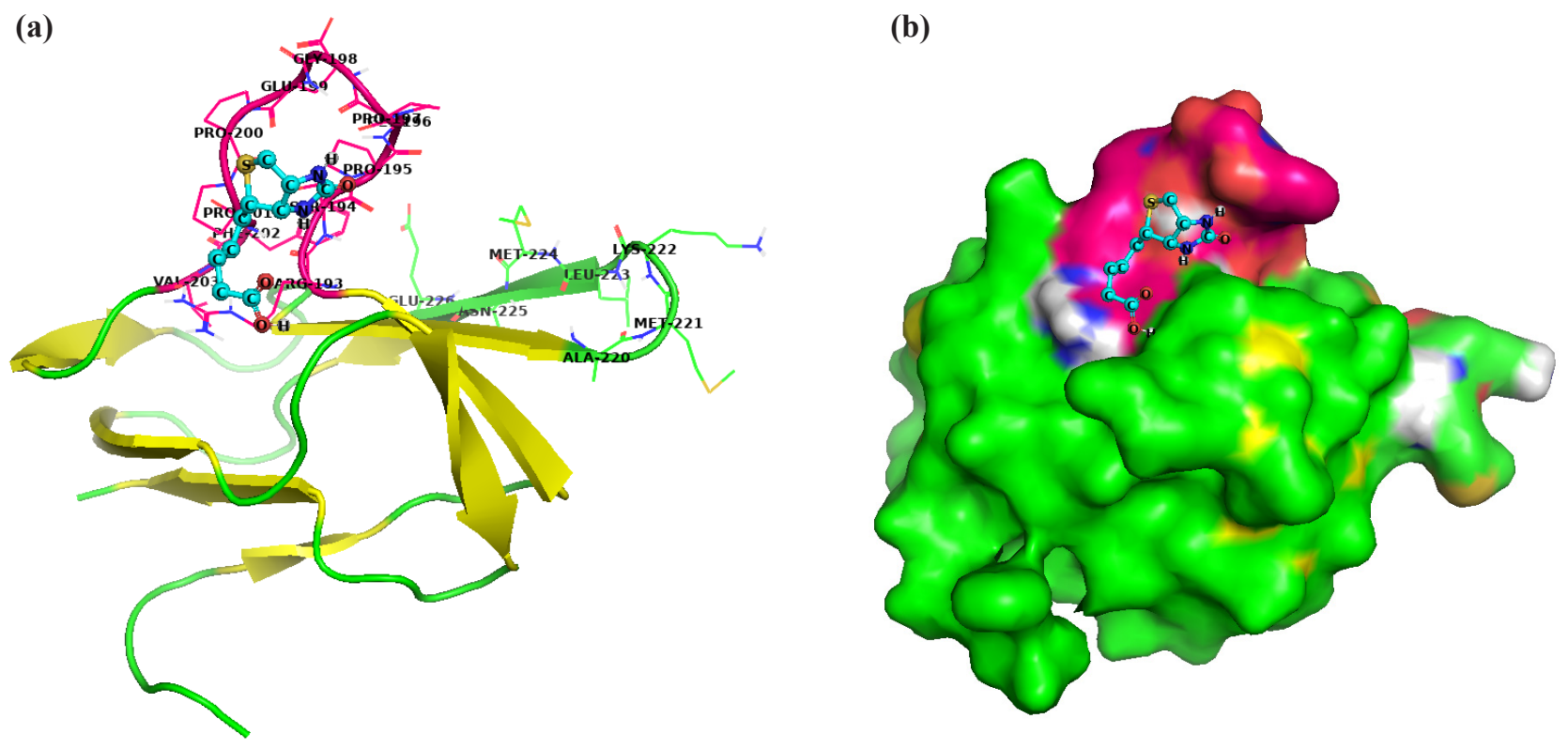

(c)

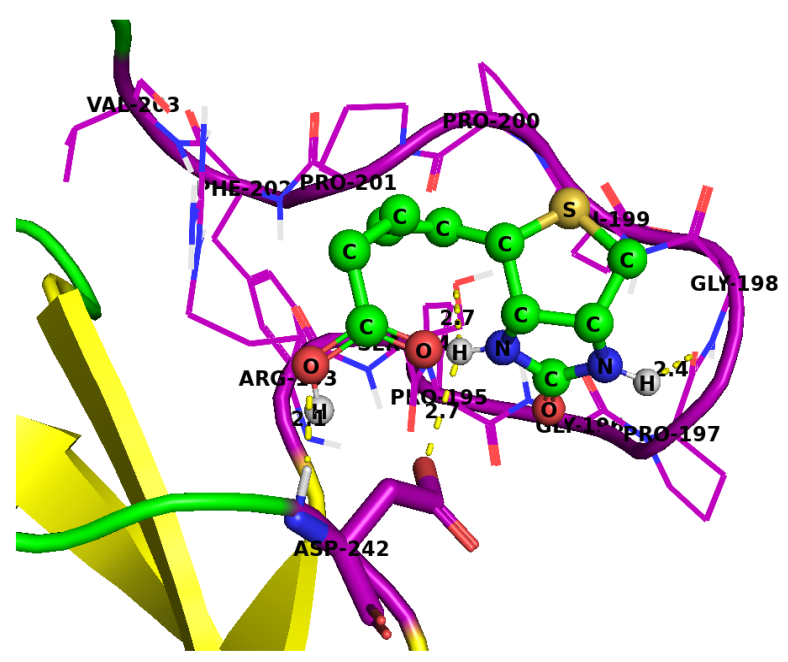

Figure 7. Molecular architecture of Br_BCCP docked with biotin molecule:The surface view of the Br_BCCP model docked with biotin (in cyano color) in its active site is depicted and the thumb region of biotinyl domain is highlighted in pink color. Active site residues of the thumb region interacting with biotin are highlighted in Figure 7. 
To interrogate the putative binding site of biotin in Br_BCCP, we did a molecular docking study of biotin with Br $\mathrm{BCCP}$ as ligand and receptor, respectively. The docked pose of biotin in Br_BCCP is depicted in Figure 7. The role of the "AMKLM " biotinylation motif in harboring biotin through the covalent bonding is reported. ${ }^{43}$ In line with this, in our investigation, we found a consensus motif in the modeled Br_BCCP protein. The ureido ring(-N-CO-N-) of biotin interacts with residues of a loop region (192 to 203) of Br_BCCP called "thumb region" and residues from $\beta$-sheets. The biotin group makes hydrogen bonds with Pro197 and Ser 194 of the thumb region and with Asp 242 of the $\beta$-sheet region at a distance of $2.4 \AA, 2.7 \AA$, and $2.7 \AA$ respectively.

(a)

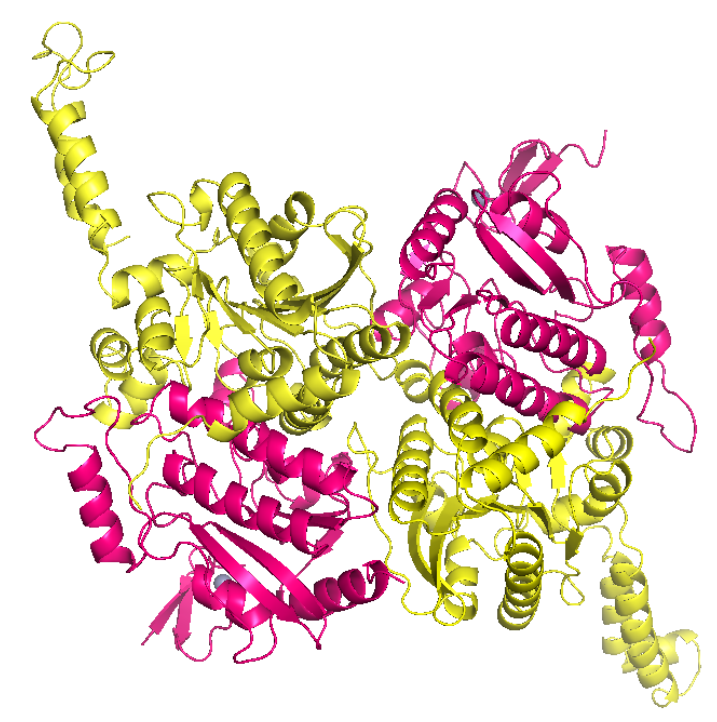

(c)

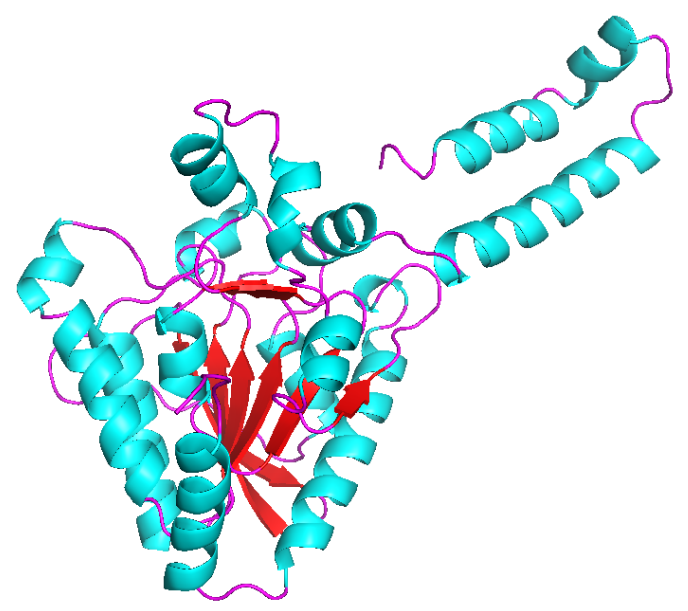

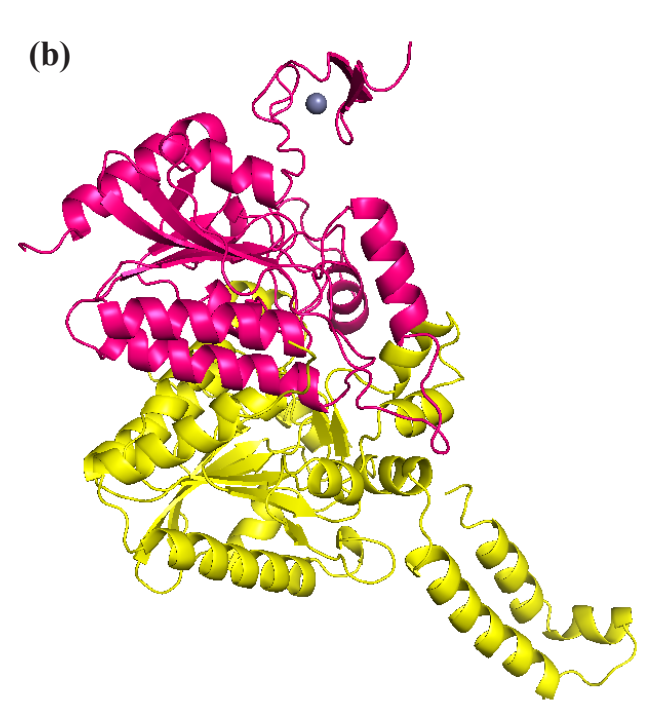

(d)

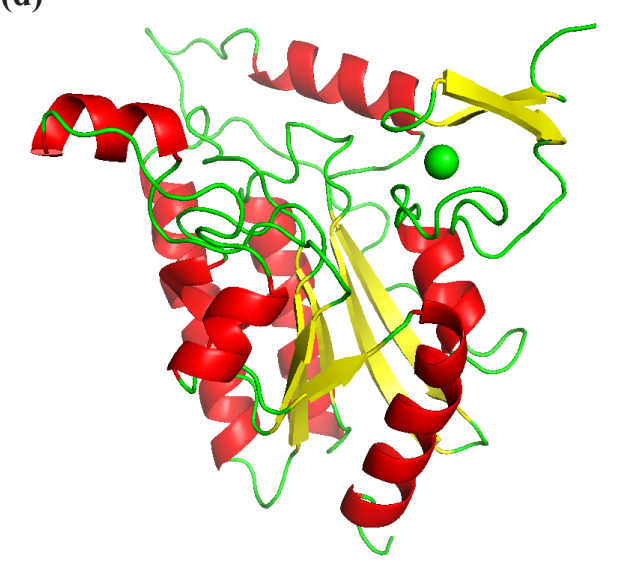

Figure 8. Homology model of Brassica rapa CT unit:Hetero-dimeric model of $\mathrm{Br}$ CT was built using 2F9I.1.pdb as a template. The model consists of CT- $\alpha$ (yellow color) and CT- $\beta$ (hot pink color) subdomains shown in Figure 8. The monomeric unit of CT showing CT- $\alpha$ (yellow) and CT- $\beta$ (hot pink) is depicted in Figure 8. Zinc ion position in CT- $\beta$ is depicted in the slate-colored sphere. The individual CT- $\alpha$ and CT- $\beta$ subdomains are depicted in Figure 9 and Figure 12, respectively showing their characteristic $\beta-\beta-\alpha$ superhelix fold.

\subsection{Molecular modeling of Brassica rapa carboxyl transferase (Br_CT) subunit of ACC}

ACC is a heterotrimeric complex consisting of a biotin carboxylase unit, biotin carboxyl carrier protein, and a carboxyl transferase (CT) unit. CT consists of two subdomains namely CT- $\alpha$ and CT- $\beta$ at its C-terminal and N-terminal, respectively. In this investigation, we built the 3D model of both CT- $\alpha$ and CT- $\beta$ of Br_CT using the crystal structure of 
carboxyl transferase subunit of ACC from Staphylococcus aureus (2F9I.1.pdb) as a "template" having 48.18\% sequence identity with the target(Br_CT) using SWISS-model server. The developed 3D model of both $\alpha$ and $\beta$ subdomain of Br_CT was evaluated for stereo-chemical quality parameters using various structure validation tools is summarized in Table-1. Results from structural and stereochemical quality parameters indicated a high degree of accuracy and reliability of the built 3D-model of both CT- $\alpha$ and CT- $\beta$ of Br_CT. Both CT- $\alpha$ and CT- $\beta$ subunit of Br_CT consists of a $\beta-\beta-\alpha$ superhelix fold which is a typical topology seen in the members of the crotonase superfamily. ${ }^{11,44^{-}}$

(a)

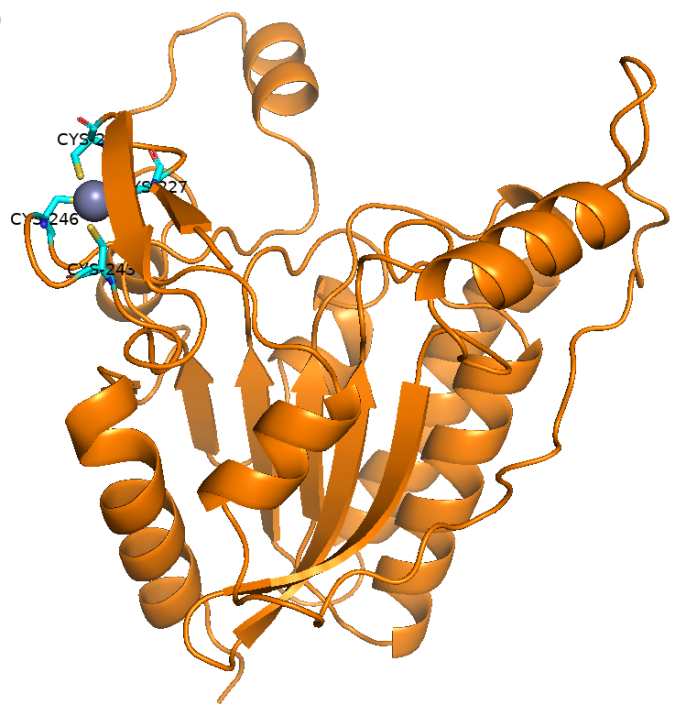

(b)

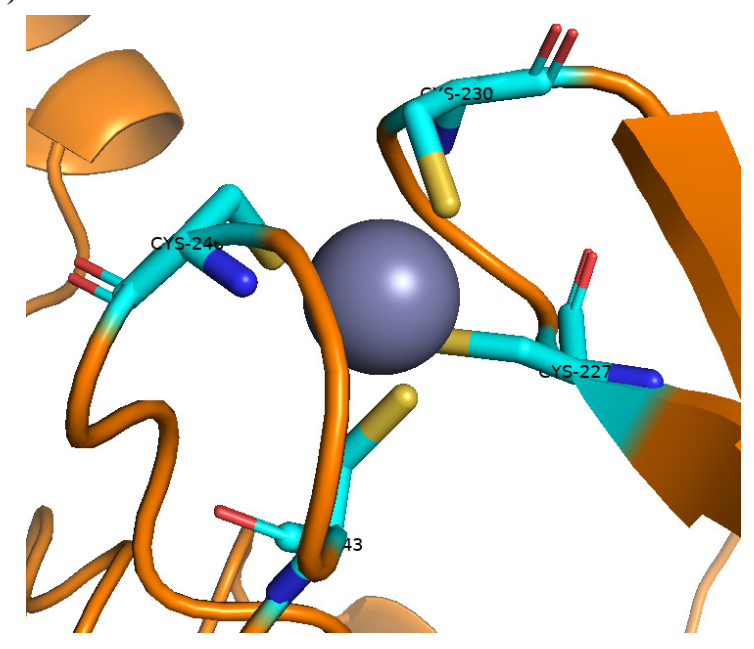

(c)

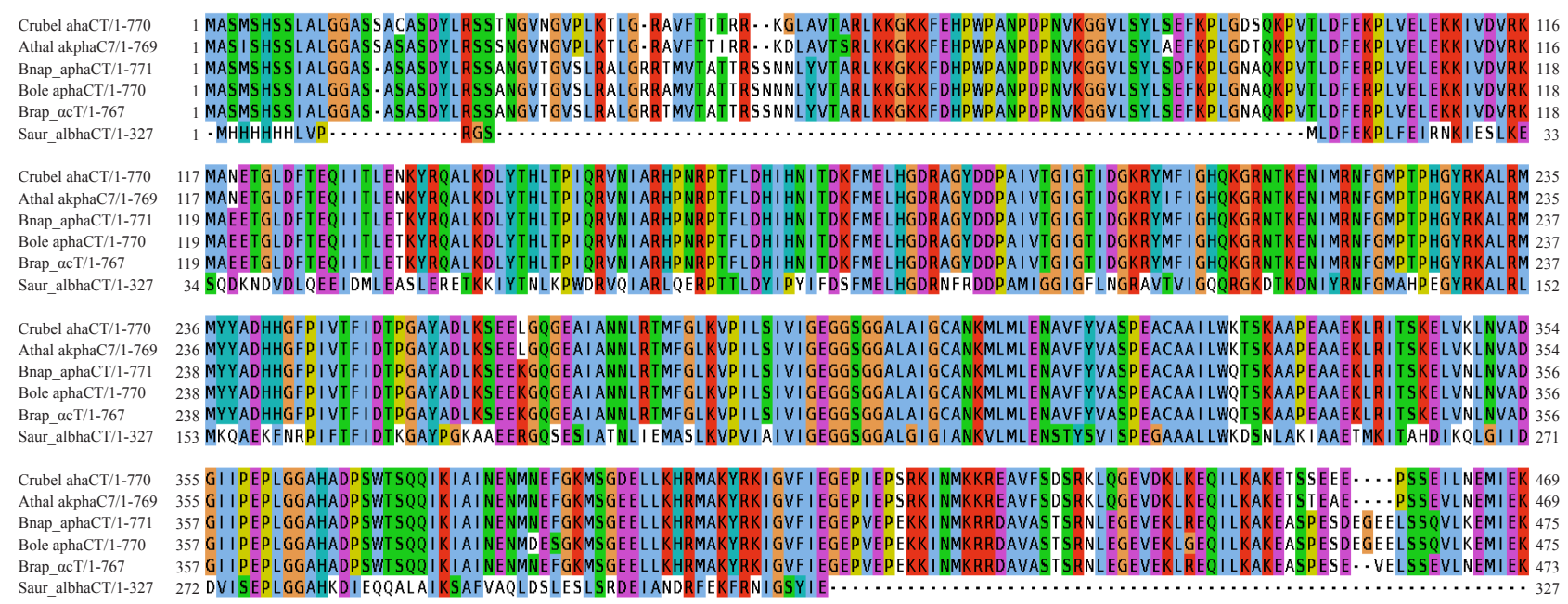

Figure 9. Visualization of zinc finger motif in CT- $\beta$ subunit in Brassica rapa \& multiple sequence alignment of CT- $\alpha$ : (a) The presence of the zincfinger motif marked by four cysteine residues coordinating with a single zinc atom in the CT- $\beta$ subunit of Brassica rapa is shown. (b) Magnified view of zinc finger motif is depicted. (c) Conservation of sequence homology among different species of plants and microbes for CT- $\alpha$ subunit is depicted in the form of a multiple sequence alignment. Crubella: Capsella rubella; Athal: Arabidopsis thaliana; Bnap: Brassica napus; Bole: Brassica oleraceae;

Brap: Brassica rapa and Saur: Staphylococcus aureus.

The core of both CT- $\alpha$ and CT- $\beta$ is composed of a six-stranded mixed $\beta$-sheet orthogonal to a two-stranded $\beta$ platform. Multiple sequence alignment analysis of CT- $\beta$ subunits of different Brassica species, Arabidopsis, and microbes revealed the presence of a strictly conserved zinc domain (CX2 CX15 CX2 C) which is a common cysteine 
four 'zinc ribbon' motif found in a wide range of proteins including ribosomal proteins, RNA polymerase II and the basal transcription factors (Figure 10). ${ }^{45}$ The exact function of the zinc domain is not known but it has been proposed that it plays a role in providing shelter to the binding site of acetyl-CoA. ${ }^{45}$ Zinc-motif is reported to play a role in the enzymatic activity of CT as its abrogation leads to complete loss of enzymatic activity while mutagenesis of cysteine residues of zinc-motif causes loss of enzymatic activity (Figure 8-Figure 10). ${ }^{46}$

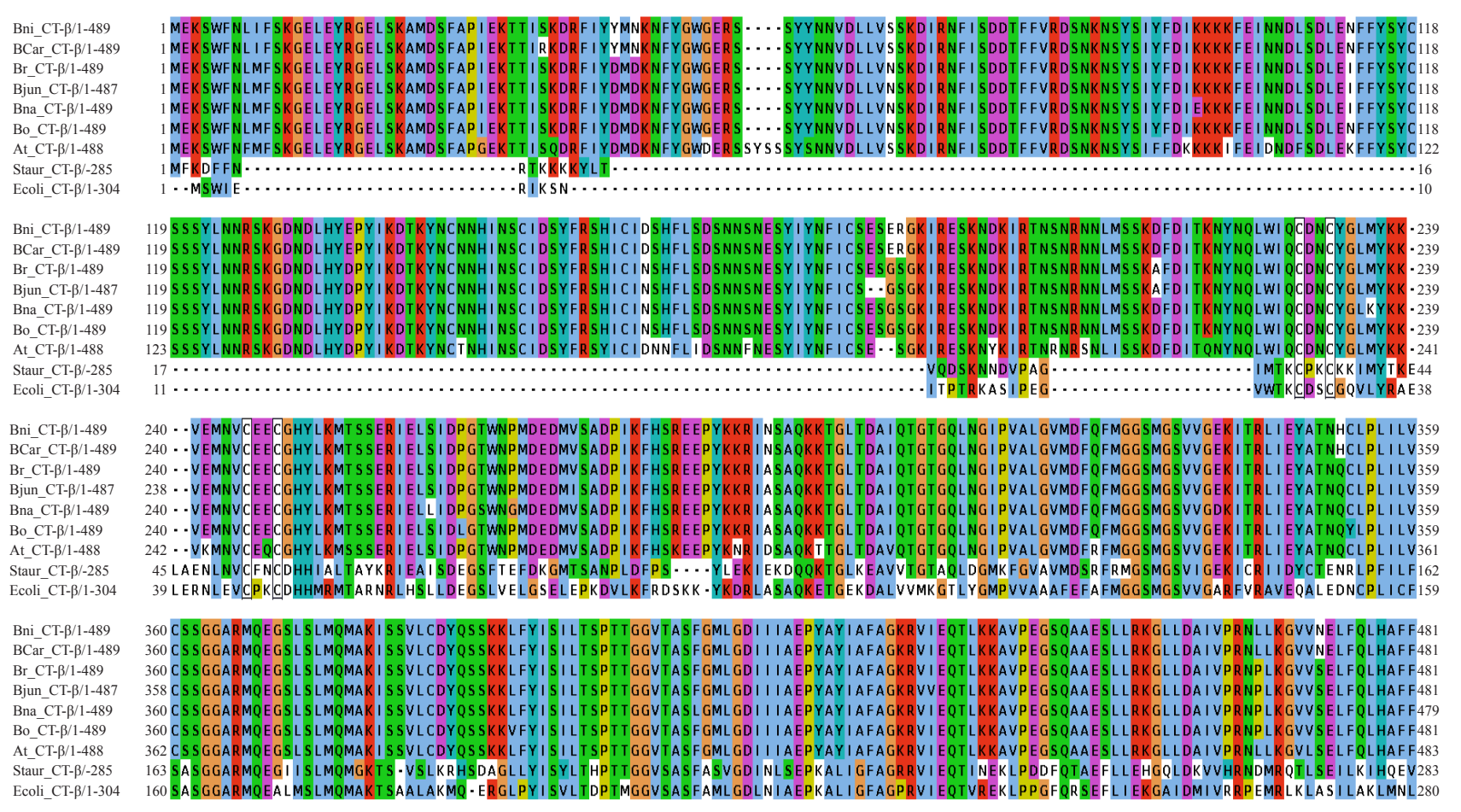

Figure 10. Multiple sequence alignment of the CT- $\beta$ subunit of ACC: The multiple sequence alignment of the $\beta$-carboxyltransferase subunit of different Brassica species along with Arabidopsis thaliana, E. coli, and Staphylococcus aureus is presented. Conserved zinc-binding sites are highlighted in boxes. Bni: Brassica nigra, Bcar: Brassica carinata, Br: Brassica rapa, Bjun: Brassica juncea, Bna: Brassica napus, Bo: Brassica oleracea, At: Arabidopsis thaliana, Staur: Staphylococcus aureus, and Ecoli: Escherichia coli.

\subsubsection{Analysis of active site of biotin carboxylase subunit of ACCase in Brassica rapa revealed evolutionary conservation of residues involved in the binding of ATP}

The acetyl-CoA and biotin-binding sites in the CT is previously reported ${ }^{45}$ in Staphylococcus aureus in which binding site for biotin was found in $\alpha$-subunit of CT at C-terminal while the acetyl-CoA binding site was located in $\beta$-subunit of CT at N-terminal just closer to the zinc-binding motif in the CT- $\beta .^{45}$ Based on the high degree of conservation between the template $S$. aureus and target CT of Brassica rapa, it can be hypothesized that the substratebinding sites might be conserved in these structures.

To prove and validate our hypothesis, we did molecular docking studies to determine the binding pose of the substrates (acetyl-CoA and biotin) in the CT domain. Analysis of best-docked conformation of acetyl-CoA with Br_CT$\beta$, it was concluded that residues namely GLY361, ALA363, GLN366, THR402, GLY404, PHE426, and GLY428 are interacting with acetyl-CoA by forming hydrogen bonds while PHE325 formed a hydrophobic interaction with acetylCoA (Figure 11). 
(a)

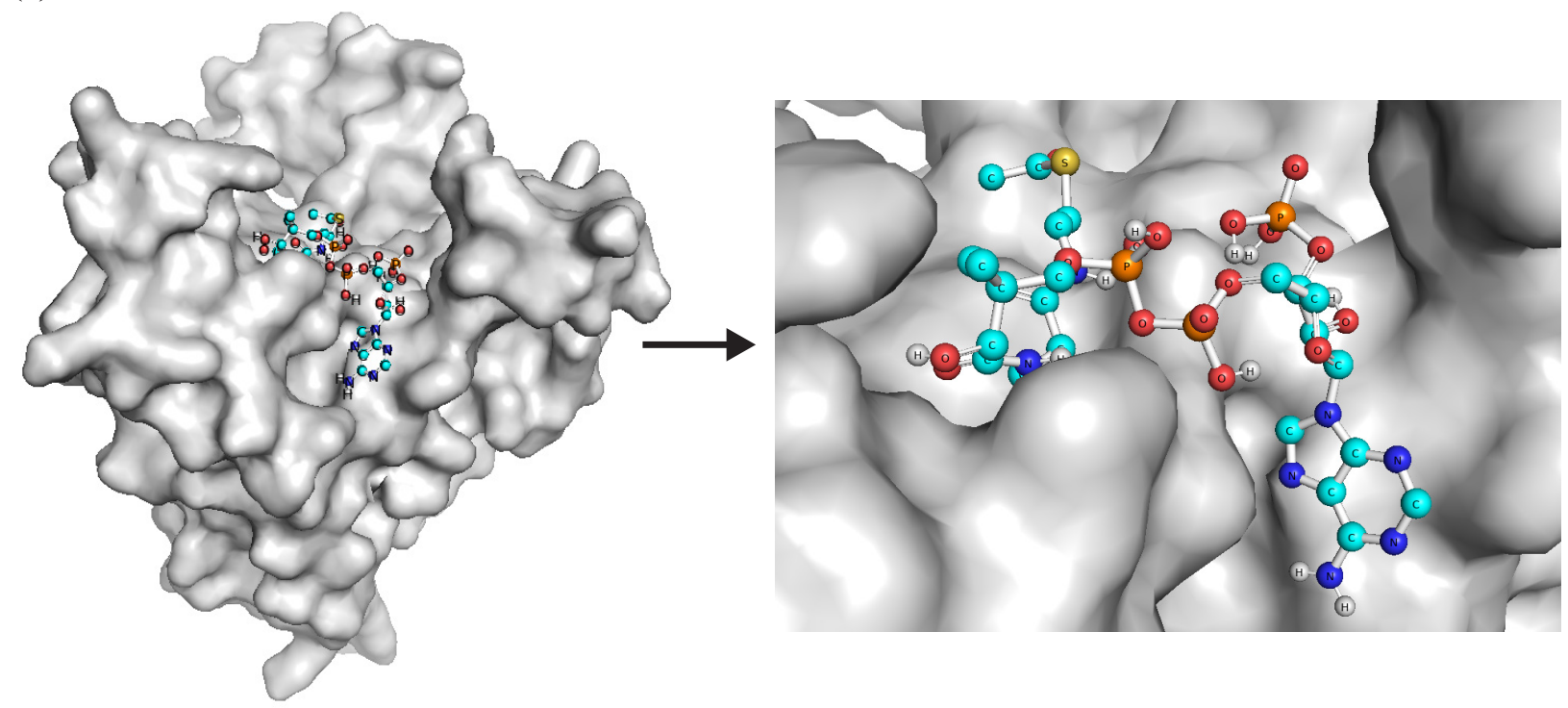

(b)

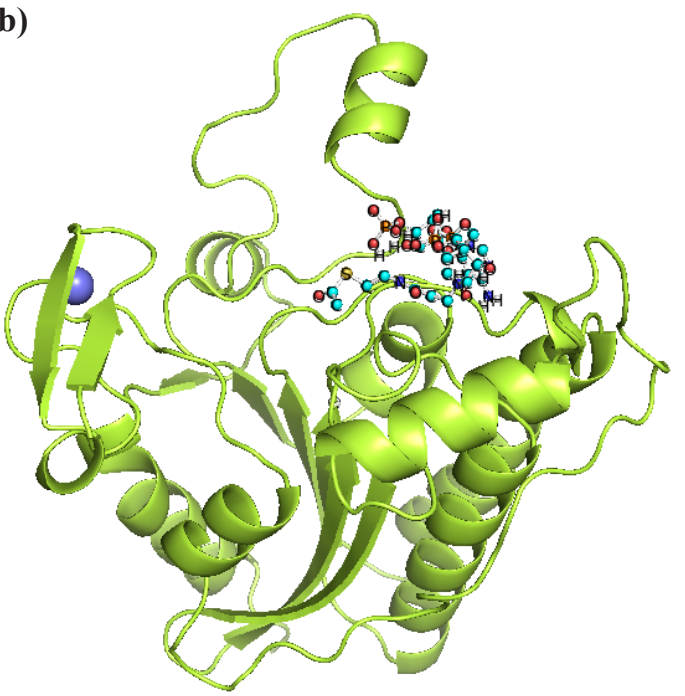

(c)

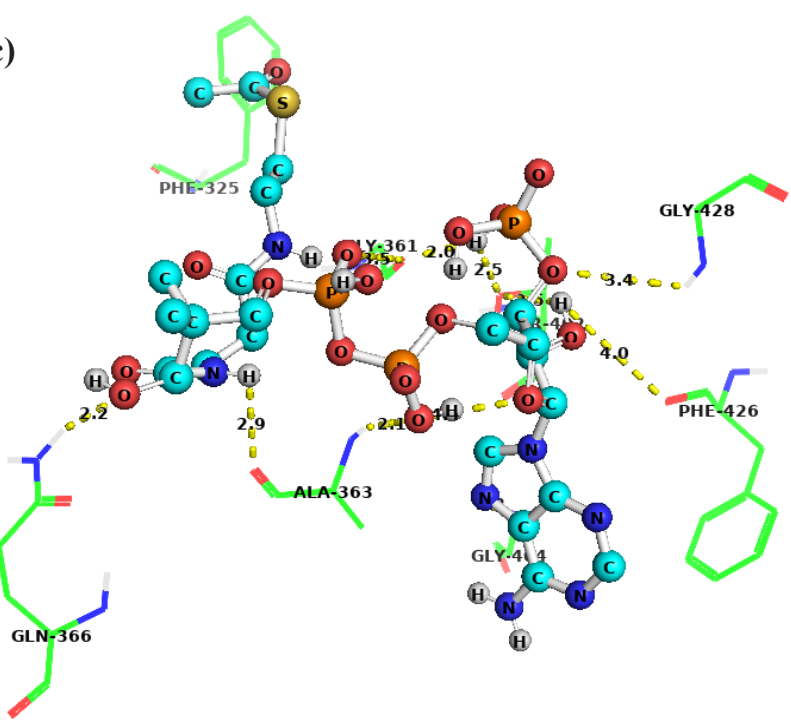

Figure 11. Visualization of acetyl-CoA binding sites in the CT- $\beta$ model in Brassica rapa: The best-docked conformation of acetyl-CoA in the active site of $\beta$ carboxyl transferase subunit (CT- $\beta$ ) is depicted in panel-(a) surface view, active site zoomed; (b) cartoon view; The active site residues interacting with acetyl-CoA are shown in panel-(c).

Similarly, a docking study between biotin and CT- $\alpha$ revealed the positioning of biotin substrate in a cavity of CT- $\alpha$ lined by residues namely ARG213, ASN262, ASP168, ASN172, PRO370, and TRP372. ARG213 and ASP168 residues interact with the imidazole group of biotin by forming hydrogen bonds. Similarly, ASN172 forms a hydrogen bond interaction with the oxygen atom of the valeric acid which is attached to the tetrahydrothiophene ring of the biotin. ASP168 and PRO370 stabilize biotin molecules by forming hydrophobic interaction (Figure 12). 
(a)

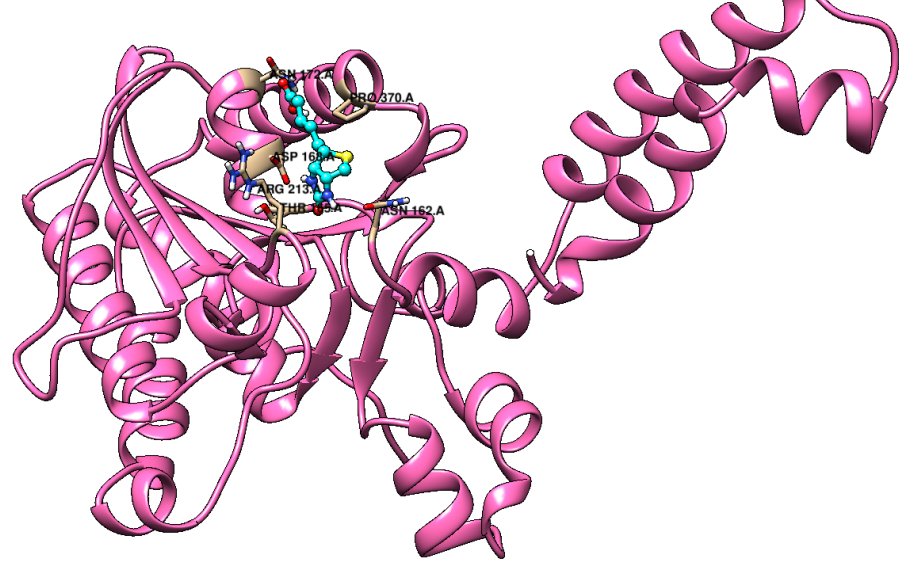

(b)

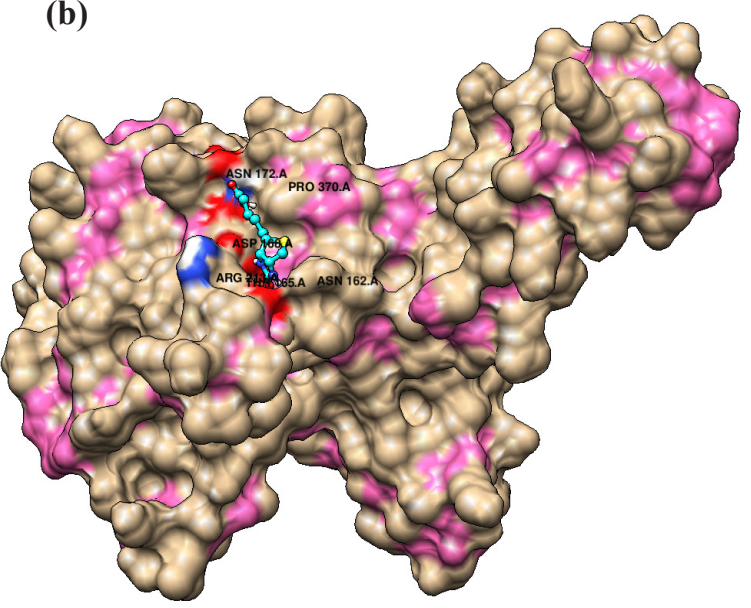

(c)

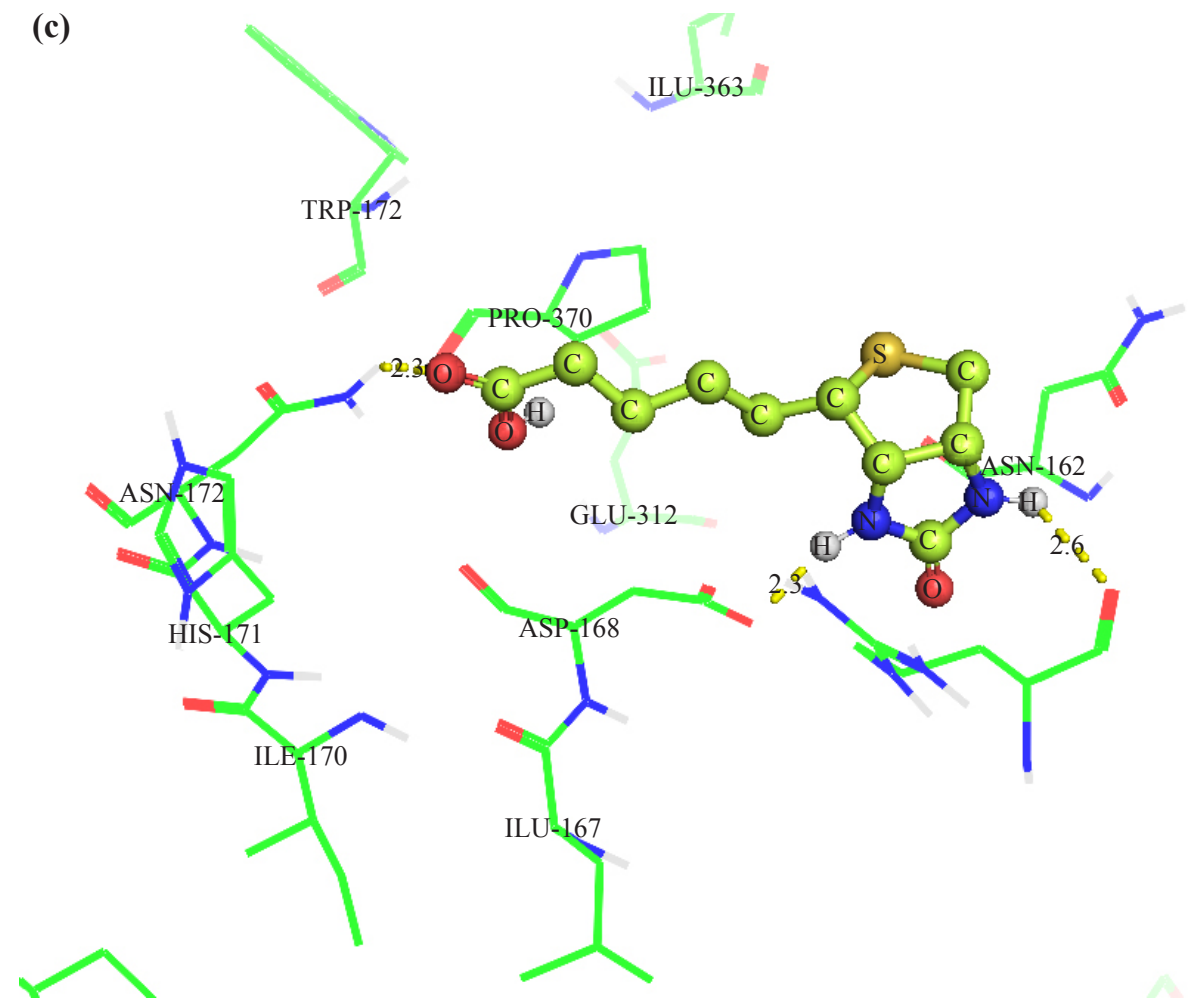

Figure 12. Visualization of biotin-binding sites in the CT- $\alpha$ model in Brassica rapa: The best-docked conformation of biotin in the active site of $\alpha$ carboxyl transferase subunit (CT- $\alpha$ ) is depicted in panel-(a)cartoon view; (b) surface view with active sites; The active site residues interacting with biotin are shown in panel-(c).

\section{Conclusions}

Expanding carbon footprints and carbon emissions due to accelerating urbanization \& industrialization drive, dependency syndrome on chemical fertilizers in intensive agriculture are the major drivers of climate change that adversely affect the growth and crop yield. Therefore, there is a need for developing innovative climate-resilient 
interventions for enhancing productivity and crop quality under climate change scenarios from a limited set of resources for food \& nutritional security of burgeoning populations. In this investigation, the molecular architecture of different components (biotin carboxylase, biotin-carboxyl-carrier protein, carboxyl transferase) of acetyl-coenzyme carboxylase of Brassica rapa is computationally predicted using homology modeling based approaches and validated for the accuracy and correctness of models using structure validation tools namely PROCHECK, ProSA, VERIFY3D, and ERRAT. Developed models were found to be reliable and accurate in terms of their structural and stereochemical attributes. Further, corresponding substrate binding sites in these models were predicted using molecular docking studies with the help of AutoDock. Based upon the molecular docking results, ATP binding sites in biotin carboxylase, biotin-binding sites in biotin carboxyl carrier protein \& in the $\alpha$-subunit of carboxyl transferase component, and acetylcoenzyme-A binding site in the $\beta$-subunit of carboxyl transferase of ACC in Brassica rapa have been predicted. All these findings on the molecular architecture of different components of ACC in Brassica rapa and ligand binding sites of component proteins from the molecular docking studies will help in two different ways. Firstly, structural information of model would facilitate designing of site-directed mutagenesis based functional genomic studies for comprehending the putative role of ACC in fatty acid biosynthesis, regulation of ACC by light \& other molecular players reported in other species such as CTI \& PII proteins. Further, functional haplotype markers can be designed using active site information of ACC in Brassica rapa to improve oil content by amalgamating desired set of available genomic variations present in different cultivars and landraces using the molecular breeding programme and genome editing tools. Such findings are potential drivers for minimizing carbon footprints by sequestering carbon in carbon skeletons of fatty acids in minimal input requiring oil-producing crop plants (Brassica rapa) without jeopardizing the ecological equilibrium.

\section{Acknowledgments}

The author expresses his/her thanks to the contributions of CKD (for literature collection, designing \& execution and manuscript preparation), UN (proofreading, manuscript preparation) and PP (for contributing to the role of Brassica rapa in carbon sequestration), and acknowledges the valuable suggestions from the anonymous peer reviewers for further improving the quality of this manuscript.

\section{Conflict of interest statements}

There are no conflict of interests among the authors.

\section{References}

[1] Change, I. P. O. C. Climate change. IPCC 2014.

[2] Solomon, S. Climate change the physical science basis; InAgu fall meeting abstracts, 2007; pp U43D-01.

[3] Zentner, R. P.; Lafond, G. P.; Derksen, D. A.; Nagy, C. N.; Wall, D. D.; May, W. E. Effects of tillage method and crop rotation on non-renewable energy use efficiency for a thin Black Chernozem in the Canadian Prairies. Soil and Tillage Res. 2004, 77, 125-136.

[4] Krupinsky, J. M.; Bailey, K. L.; McMullen, M. P.; Gossen, B. D.; Turkington, T. K. Managing plant disease risk in diversified cropping systems. Agron. J. 2002, 94, 198-209.

[5] Gan, Y.; Liang, C.; Hamel, C.; Cutforth, H.; Wang, H. Strategies for reducing the carbon footprint of field crops for semiarid areas. Agron. for Sust. Dev. 2011, 31, 643-656.

[6] Gan, Y.; Liang, C.; Wang, X.; McConkey, B. Lowering carbon footprint of durum wheat by diversifying cropping systems. Field Crops Res. 2011, 122, 199-206.

[7] Lee, J. S.; Abood, S.; Ghazoul, J.; Barus, B.; Obidzinski, K.; Koh, L. P. Environmental impacts of large scale oil palm enterprises exceed that of smallholdings in Indonesia. Conservation lett. 2014, 7, 25-33.

[8] Vijay, V.; Pimm, S. L.; Jenkins, C. N.; Smith, S. J. The impacts of oil palm on recent deforestation and biodiversity loss. PloS one. 2016, 11, e 0159668.

[9] Smith, E. G.; Janzen, H. H.; Newlands, N. K. Energy balances of biodiesel production from soybean and canola in 
Canada. Canadian J. Pl. Sci. 2007, 87, 793-801.

[10] Singh, R.; Malhotra, S. P. Carbon fixation, sucrose synthesis and its transport to storage tissues. Dev. in Crop Sci. 2000, 26, 1-34.

[11] Cronan Jr, J. E.; Waldrop. G. L. Multi-subunit acetyl-CoA carboxylases. Prog. in lipid res. 2002, 41, 407-435.

[12] Sasaki, Y.; Nagano, Y. Plant acetyl-CoA carboxylase: structure, biosynthesis, regulation, and gene manipulation for plant breeding. Biosci. biotech. and biochem. 2004, 68, 1175-1184.

[13] Broussard, T. C.; Kobe, M. J.; Pakhomova, S.; Neau, D. B.; Price, A. E.; Champion, T. S.; Waldrop, G. L. The three-dimensional structure of the biotin carboxylase-biotin carboxyl carrier protein complex of E. coli acetyl-CoA carboxylase. Structure. 2013, 21, 650-657.

[14] Harwood, J. L.; Walsh, M. C.; Walker, K. A. Methods in Plant Biochemistry; Academic Press, London, 1990; pp 96-110.

[15] Davis, M.S.; Solbiati, J.; Cronan Jr, J. E. Overproduction of acetyl-CoA carboxylase activity increases the rate of fatty acid biosynthesis in Escherichia coli. J. of Bio. Chem. 2000, 275, 28593-28598.

[16] Menendez, C.; Bauer, Z.; Huber, H.; Gad'on, N.; Stetter, K. O.; Fuchs, G. Presence of acetyl coenzyme A (CoA) carboxylase and propionyl-CoA carboxylase in autotrophic Crenarchaeota and indication for operation of a 3-hydroxypropionate cycle in autotrophic carbon fixation. J. of Bact. 1999, 181, 1088-1098.

[17] Ye, Y.; Fulcher, Y. G.; Sliman, D. J.; Day, M. T.; Schroeder, M. J.; Koppisetti, R. K.; Bates, P. D.; Thelen, J. J.; Van Doren, S. R. The BADC and BCCP subunits of chloroplast acetyl-CoA carboxylase sense the pH changes of the light-dark cycle. J. of Bio. Chem. 2020, 295, 9901-9916.

[18] Andre, C.; Haslam, R. P.; Shanklin, J. Feedback regulation of plastidic acetyl-CoA carboxylase by 18:1-acyl carrier protein in Brassica napus. Proc. of the National Acad. of Sci. 2012, 109, 10107-10112.

[19] Bourrellier, A. B.; Valot, B.; Guillot, A.; Ambard-Bretteville, F.; Vidal, J.; Hodges, M. Chloroplast acetyl-CoA carboxylase activity is 2-oxoglutarate-regulated by interaction of PII with the biotin carboxyl carrier subunit. Proc. of the National Acad. of Sci. 2010, 107, 502-507.

[20] Shivaiah, K. K.; Ding, G.; Upton, B.; Nikolau, B. J. Non-catalytic subunits facilitate quaternary organization of plastidic acetyl-CoA carboxylase. Plant Phys. 2020, 182, 756-775.

[21] Elborough, K. M.; Winz, R.; Deka, R. K.; Markham, J. E.; White, A. J.; Rawsthorne, S.; Slabas, A. R. Biotin carboxyl carrier protein and carboxyltransferase subunits of the multi-subunit form of acetyl-CoA carboxylase from Brassica napus: cloning and analysis of expression during oilseed rape embryogenesis. Biochem. J. 1996, 315, 103112.

[22] Zhang, Z.; Dunwell, J. M.; Zhang, Y. M. An integrated omics analysis reveals molecular mechanisms that are associated with differences in seed oil content between Glycine max and Brassica napus. BMC plant bio. 2018, 18 , $1-5$.

[23] Hu, Y.; Wu, G.; Cao, Y.; Wu, Y.; Xiao, L.; Li, X.; Lu, C. Breeding response of transcript profiling in developing seeds of Brassica napus. BMC Mol. Bio. 2009, 10, 1-7.

[24] Mochalkin, I., Miller, J. R.; Evdokimov, A.; Lightle, S.; Yan, C.; Stover, C. K.; Waldrop, G. L. Structural evidence for substrate induced synergism and half sites reactivity in biotin carboxylase. Protein Sci. 2008, 17, $1706-1718$.

[25] Waterhouse, A.; Bertoni, M.; Bienert, S.; Studer, G.; Tauriello, G.; Gumienny, R.; Heer, F. T.; de Beer, T. A.; Rempfer, C.; Bordoli, L.; Lepore, R. SWISS-MODEL: homology modelling of protein structures and complexes. Nucleic acids res. 2018, 46, W296-W303.

[26] Sippl, M. J. Recognition of errors in three dimensional structures of proteins. Proteins: Structure, Function, and Bioinformatics. 1993, 17, 355-362.

[27] Wiederstein, M.; Sippl, M. J. ProSA-web: interactive web service for the recognition of errors in three-dimensional structures of proteins. Nucleic acids res. 2007, 35, W407-W410.

[28] Laskowski, R. A.; MacArthur, M. W.; Moss, D. S.; Thornton, J. M. PROCHECK: A program to check the stereochemical quality of protein structures. J. of app. cryst. 1993, 26, 283-291.

[29] Eisenberg, D.; Lüthy, R.; Bowie, J. U. VERIFY3D: assessment of protein models with three-dimensional profiles. Methods in enz. 1997, 277, 396-404.

[30] Colovos, C.; Yeates, T. O. Verification of protein structures: patterns of nonbonded atomic interactions. Protein Sci. 1993, 2, 1511-1519.

[31] Trott, O.; Olson, A. J. AutoDock Vina: improving the speed and accuracy of docking with a new scoring function, efficient optimization, and multithreading. J. of comp. chem. 2010, 31, 455-461.

[32] Wang, Y.; Bolton, E.; Dracheva, S.; Karapetyan, K.; Shoemaker, B. A.; Suzek, T. O.; Wang, J.; Xiao, J.; Zhang, J.; Bryant, S. H. An overview of the PubChem BioAssay resource. Nucleic acids res. 2010, 38, D255-D266.

[33] Pettersen, E. F.; Goddard, T. D.; Huang, C. C.; Couch, G. S.; Greenblatt, D. M.; Meng, E. C.; Ferrin, T. E. UCSF 
Chimera-a visualization system for exploratory research and analysis. J. of comp. chem. 2004, 25, 1605-1612.

[34] DeLano, W. L. Pymol: An open-source molecular graphics tool. CCP4 News. on protein cryst. 2002, 40, 82-92.

[35] Jeanmougin, F.; Thompson, J. D.; Gouy, M.; Higgins, D. G.; Gibson, T. J. Multiple sequence alignment with Clustal X. Trends in biochem. sci. 1998, 23, 403-405.

[36] Waterhouse, A. M.; Procter, J. B.; Martin, D. M.; Clamp, M.; Barton, G. J. Jalview Version 2-a multiple sequence alignment editor and analysis workbench. Bioinformatics. 2009, 25, 1189-1191.

[37] Galperin, M. Y.; Koonin, E. V. A diverse superfamily of enzymes with ATP dependent carboxylate-amine/thiol ligase activity. Protein Sci. 1997, 6, 2639-2643.

[38] Climent, I.; Rubio, V. ATPase activity of biotin carboxylase provides evidence for initial activation of $\mathrm{HCO}_{3}$ - by ATP in the carboxylation of biotin. Arch. of biochem. and biophys. 1986, 251, 465-470.

[39] Misra, N.; Panda, P. K.; Patra, M. C.; Pradhan, S. K.; Mishra, B. K. Insights into Molecular Assembly of ACCase Heteromeric Complex in Chlorella variabilis-A Homology Modelling, Docking and Molecular Dynamic Simulation Study. App. biochem. and biotech. 2013, 170, 1437-1457.

[40] Thoden, J. B.; Blanchard, C. Z.; Holden, H. M.; Waldrop, G. L. Movement of the biotin carboxylase B-domain as a result of ATP binding. J. of Bio. Chem. 2000, 275, 16183-16190.

[41] Toh, H.; Kondo, H.; Tanabe, T. Molecular evolution of biotin dependent carboxylases. Europ. J of biochem. 1993, $215,687-696$

[42] Zhu, X. L.; Zhang, L.; Chen, Q.; Wan, J.; Yang, G. F. Interactions of aryloxyphenoxypropionic acids with sensitive and resistant acetyl-coenzyme a carboxylase by homology modeling and molecular dynamic simulations. $J$. of chem. inform. and mod. 2006, 46, 1819-1826.

[43] Thelen, J. J.; Mekhedov, S.; Ohlrogge, J. B. Brassicaceae express multiple isoforms of biotin carboxyl carrier protein in a tissue-specific manner. Plant Phys. 2001, 125, 2016-2028.

[44] Holden, H. M.; Benning, M. M.; Haller, T.; Gerlt, J. A. The crotonase superfamily: divergently related enzymes that catalyze different reactions involving acyl coenzyme a thioesters. Acc. of chem. res. 2001, 34, 145-157.

[45] Bilder, P.; Lightle, S.; Bainbridge, G.; Ohren, J.; Finzel, B.; Sun, F.; Holley, S.; Al-Kassim, L.; Spessard, C.; Melnick, M.; Newcomer, M. The structure of the carboxyltransferase component of acetyl-coA carboxylase reveals a zinc-binding motif unique to the bacterial enzyme. Biochemistry. 2006, 45, 1712-1722.

[46] Kozaki, A.; Mayumi, K.; Sasaki, Y. Thiol-disulfide exchange between nuclear-encoded and chloroplast-encoded subunits of pea acetyl-CoA carboxylase. J. of Biol. Chem. 2001, 276, 39919-39925.

[47] Chapman, K. D.; Ohlrogge, J. B. Compartmentation of triacylglycerol accumulation in plants. J. of Bio. Chem. 2012, 287, 2288-2294. 